\title{
Perron's method and the method of relaxed limits for "unbounded" PDE in Hilbert spaces
}

\author{
by \\ DJivede Kelome (Amherst, MA, and Montreal) and \\ AndrzeJ Święch (Atlanta, GA)
}

\begin{abstract}
We prove that Perron's method and the method of half-relaxed limits of Barles-Perthame works for the so called $B$-continuous viscosity solutions of a large class of fully nonlinear unbounded partial differential equations in Hilbert spaces. Perron's method extends the existence of $B$-continuous viscosity solutions to many new equations that are not of Bellman type. The method of half-relaxed limits allows limiting operations with viscosity solutions without any a priori estimates. Possible applications of the method of half-relaxed limits to large deviations, singular perturbation problems, and convergence of finite-dimensional approximations are discussed.
\end{abstract}

1. Introduction. In this paper we investigate the possibility of extending Perron's method and the method of half-relaxed limits of BarlesPerthame to a class of equations in infinite-dimensional Hilbert spaces of the form

$$
u+\langle A x, D u\rangle+F\left(x, D u, D^{2} u\right)=0
$$

and their time dependent versions

$$
\left\{\begin{array}{l}
u_{t}+\langle A x, D u\rangle+F\left(t, x, D u, D^{2} u\right)=0, \quad(t, x) \in(0, T) \times H, \\
u(0, x)=g(x)
\end{array}\right.
$$

Here $H$ is a real separable Hilbert space with the inner product $\langle\cdot, \cdot\rangle$ and norm $\|\cdot\|$, and $A$ is a linear, maximal monotone operator in $H$. The symbols $D u, D^{2} u$ denote the Fréchet derivatives of $u$. This is a large class of equations that includes Hamilton-Jacobi-Bellman (HJB) equations for optimal control of stochastic semilinear PDE (for instance stochastically perturbed reaction-diffusion equations) and delay equations, Isaacs equations, infinite-dimensional Black-Scholes-Barenblatt equation for option pricing, and many others.

2000 Mathematics Subject Classification: 49L25, 35R15, 35J60.

Key words and phrases: viscosity solutions, Hamilton-Jacobi-Bellman equations, Perron's method, relaxed limits, Hilbert spaces.

A. Święch was supported by NSF grant DMS 0500270. 
There exists a good theory of such equations based on the notion of the so called $B$-continuous viscosity solution $[5,6,25]$. The theory however still lacks several key components that are among the main tools of viscosity solutions in finite-dimensions, namely Perron's method and the method of half-relaxed limits. Perron's method is the main technique for producing viscosity solutions of PDE in finite-dimensional spaces (see [3]). It is based on the principle that the supremum of the family of all viscosity subsolutions of an equation is a viscosity solution and so all we need to do to prove existence of a viscosity solution is to produce one sub- and one supersolution. Despite previous efforts it is still not known if a version of Perron's method can be implemented for $B$-continuous viscosity solutions of (1.1) and (1.2), even if the equations are of first order. Perron's method works with Ishii's definitions of solutions [17, 18] (see also [23]), but his notion of solution [18] does not seem easily applicable to stochastic optimal control problems and is not used. Half-relaxed limits of Barles-Perthame (see [3]) are perhaps an even more fundamental technique in the theory of viscosity solutions that is widely used to pass to weak limits without any a priori estimates. A huge part of the success of viscosity solutions is based on the fact that limiting operations are very easy in this framework. It is known that due to the lack of local compactness in infinite-dimensional spaces this procedure may not work in general, even for simple equations with $A=0$ (see $[1,26]$ ).

In this paper we will show that both Perron's method and the method of half-relaxed limits can be adapted for equations (1.1) and (1.2) if the operator $A$ satisfies a coercivity condition (2.2). It has been noticed before $[2,8,13-16]$ that a condition of this type leads to a stronger definition of viscosity solution and this stronger definition will help us overcome technical difficulties needed to implement both methods.

Apart from providing an easy method to produce solutions another consequence of Perron's method will be new existence results for a large class of equations of the above types. Currently there exist two methods for proving existence of $B$-continuous viscosity solutions of (1.1) and (1.2): by finitedimensional approximations [25], and by using stochastic analysis to show that the value function of the associated stochastic optimal control problem solves the PDE $[13,15,16,19]$. The first is limited to the case of the operator $B$ (see Section 2) being compact, and the second to HJB equations related to optimal control problems. Perron's method will allow one to construct solutions for general operators $B$ and equations that are not of Bellman type, for instance for Isaacs equations related to stochastic differential games. The method of half-relaxed limits should have significant impact on the theory of PDE in Hilbert spaces, especially since passing to limits in infinite-dimensional spaces is very difficult even with good a priori estimates. Moreover we anticipate many other interesting applications, for instance in 
the theory of large deviations and risk sensitive control. In these problems one has to deal with small noise limits that correspond to singular perturbation problems for the associated HJB equations. For instance for diffusions driven by stochastic PDE with additive noise these HJB equations may have the form

$$
\left(u_{\varepsilon}\right)_{t}-\varepsilon \operatorname{Tr}\left(Q D^{2} u_{\varepsilon}\right)+\left\langle A x, D u_{\varepsilon}\right\rangle+F\left(t, x, D u_{\varepsilon}\right)=0
$$

for some trace class operator $Q=Q^{*} \geq 0$, and one is interested in the behavior of their solutions $u_{\varepsilon}$ as $\varepsilon \rightarrow 0$. Recently Feng and Kurtz [12] proposed a very general framework for large deviations based on viscosity solutions in abstract spaces. However, they only use viscosity solutions of the limiting first order equation and the rest of the method relies on convergence of nonlinear semigroups and stochastic analysis making it a little cumbersome to apply. A similar approach is used in $[10,11]$ for Hilbert space valued diffusions. In [11] Tataru's definition of viscosity solution [27, 28, 7] is used for the limiting first order Hamilton-Jacobi equation and the passage to the limit is based on the convergence of generators and the comparison principle for the limiting Hamilton-Jacobi equation. We think that the theory of second order HJB equations is crucial to a good PDE approach to large deviations. The method of half-relaxed limits is a purely analytical technique that makes passing to singular limits almost trivial. It seems to be a perfect tool for large deviation arguments for Hilbert space valued diffusions, including exit time problems. We will present applications to large deviations in a future publication. Another possible applications of the method of half-relaxed limits include convergence results for finite-dimensional approximations that would give a "Galerkin" type procedure for (1.1) and (1.2) without any a priori estimates. Apart from its theoretical value such a method may for instance help produce numerical methods for solving infinite-dimensional equations, and may help develop procedures for constructing $\varepsilon$-optimal feedback controls. The possibilities seem wide open.

Finally, we refer the reader to [9] for an overview of the established theory of PDE in Hilbert spaces by methods other than viscosity solutions.

2. Notation and assumptions. We will always identify $H$ with its dual space. With this identification we can interpret the Fréchet derivatives $D u(x)$ and $D^{2} u(x)$ as respectively an element of $H$ and a bounded, selfadjoint operator in $H$. We will denote the space of bounded, self-adjoint operators in $H$ by $\mathcal{S}(H)$.

Throughout the paper $B$ will be a fixed bounded, positive, self-adjoint operator such that $A^{*} B$ is bounded and

$$
\left\langle\left(A^{*} B+C_{0} B\right) x, x\right\rangle \geq 0 \text { for some } C_{0}>0 \text { and all } x \in H .
$$


Such an operator always exists, for instance $B=\left(A^{*}+I\right)^{-1}(A+I)^{-1}$ or $B=\left((A+I)\left(A^{*}+I\right)\right)^{-1 / 2}$. We refer the reader to [22] for the proof of the latter and to [5] for examples of other possible $B$ in some particular cases. The operator $B$ defines spaces $H_{\alpha}$. For $\alpha<0$ we define $H_{\alpha}$ as the completion of $H$ under the norm $\|x\|_{\alpha}=\left\|B^{\alpha / 2} x\right\|$, and for $\alpha>0, H_{\alpha}=R\left(B^{\alpha / 2}\right)$ equipped with the norm $\|x\|_{\alpha}=\left\|B^{-\alpha / 2} x\right\|$. They are Hilbert spaces with the inner product $\langle x, y\rangle_{\alpha}=\left\langle B^{-\alpha / 2} x, B^{-\alpha / 2} x\right\rangle, H_{\alpha}$ and $H_{-\alpha}$ are dual to each other, and $B^{\alpha / 2}$ is an isometry between $H_{\beta}$ and $H_{\beta+\alpha}$.

We will require that $A$ satisfy the coercivity condition

$$
\left\langle A^{*} x, x\right\rangle \geq \lambda\|x\|_{1}^{2} \quad \text { for } x \in D\left(A^{*}\right)
$$

for some $\lambda>0$.

The above implies in particular that $D\left(A^{*}\right) \subset H_{1}$. This assumption is satisfied for instance for self-adjoint invertible operators $A$ if $B=A^{-1}$.

We will always assume that $F:[0, T] \times H \times H \times \mathcal{S}(H) \rightarrow \mathbb{R}$ is locally uniformly continuous and is degenerate elliptic, i.e.

$$
F(t, x, p, X) \geq F(t, x, p, Y) \quad \text { when } X \leq Y .
$$

Let $\left\{e_{1}, e_{2}, \ldots\right\}$ be a basis of $H_{-1}$ consisting of elements of $H$. Given $N \geq 1$ let $V_{N}=\operatorname{span}\left\{e_{1}, e_{2}, \ldots, e_{N}\right\}$, and let $P_{N}$ denote the orthogonal projection of $H_{-1}$ onto $V_{N}$. Denote $Q_{N}=I-P_{N}$ where $I$ is the identity in $H_{-1}$. We will sometimes need several additional conditions on the Hamiltonian $F$. Let $k \geq 0$.

$(\mathbf{1})_{k}$ There exists a radial function $\mu(x)=\mu(\|x\|)$ satisfying the conditions in Definition 3.1(iii) below and $K>0$ such that

$$
\lim _{\|x\| \rightarrow \infty} \frac{\mu(x)}{\|x\|^{k}}=\infty
$$

and for every positive real number $\alpha, t \in[0, T], x, p \in H, X \in \mathcal{S}(H)$,

$$
\left|F\left(t, x, p+\alpha D \mu(x), X+\alpha D^{2} \mu(x)\right)-F(t, x, p, X)\right| \leq K \alpha(1+\mu(x)) .
$$

(2) For all $t \in[0, T], x, p \in H, R>0$, $\sup \left\{\left|F\left(t, x, p, X+\lambda B Q_{N}\right)-F(t, x, p, X)\right|:\|X\|,|\lambda| \leq R, P_{N}^{*} X P_{N}=X\right\} \rightarrow 0$ as $N \rightarrow \infty$.

(3) There exist moduli $\omega_{R}$ such that

$$
\begin{aligned}
F(t, x, B(x-y) / \varepsilon, X)-F(t, & y, B(x-y) / \varepsilon,-Y) \\
& \geq-\omega_{R}\left(\|x-y\|_{-1}\left(1+\|(x-y)\|_{-1} / \varepsilon\right)\right)
\end{aligned}
$$

whenever $\|x\|,\|y\| \leq R$ and $X, Y$ satisfy the inequality

$$
\left(\begin{array}{cc}
X & 0 \\
0 & Y
\end{array}\right) \leq \frac{2}{\varepsilon}\left(\begin{array}{cc}
B P_{N} & -B P_{N} \\
-B P_{N} & B P_{N}
\end{array}\right) .
$$


We point out that condition (3) can be weakened if $A$ and $B$ satisfy a stronger version of (2.1), namely if

$$
\left\langle\left(A^{*} B+C_{0} B\right) x, x\right\rangle \geq\|x\|^{2} \quad \text { for some } C_{0}>0 \text { and all } x \in H \text {. }
$$

According to [22], (2.3) is always satisfied for $B=\left((A+I)\left(A^{*}+I\right)\right)^{-1 / 2}$ if $(2.2)$ holds, and $[D(A), H]_{1 / 2}=\left[D\left(A^{*}\right), H\right]_{1 / 2}=H_{1}$, where $[\cdot, \cdot]_{1 / 2}$ is the interpolation space (see [21]). Also it is easy to see that (2.3) holds if (2.2) holds and for instance if $\left\|\left(B^{1 / 2} A^{*} B^{1 / 2}-A^{*} B\right) x\right\| \leq C_{1}\|x\|_{-1}$. To see this it is enough to notice that

$$
\left\langle A^{*} B x, x\right\rangle \geq\left\langle B^{1 / 2} A^{*} B^{1 / 2} x, x\right\rangle-C_{2}\|x\|_{-1}\|x\| \geq \frac{\lambda}{2}\|x\|^{2}-C_{2}\|x\|_{-1}^{2} .
$$

In particular, (2.3) holds if $A$ is self-adjoint and invertible and $B=A^{-1}$. However we will not state any results for the stronger case (2.3).

We will say that a function $u:[0, T] \times H \rightarrow \mathbb{R}$ is $B$-upper semicontinuous (respectively, B-lower semicontinuous) if whenever $t_{n} \rightarrow t$ and $\left\|x_{n}-x\right\|_{-1} \rightarrow 0$ for a bounded sequence $x_{n}$ then $\limsup _{n \rightarrow \infty} u\left(t_{n}, x_{n}\right) \leq u(t, x)$ (respectively, $\left.\liminf _{n \rightarrow \infty} u\left(t_{n}, x_{n}\right) \geq u(t, x)\right)$. A function is $B$-continuous if it is both $B$-upper semicontinuous and $B$-lower semicontinuous. A function is $l o-$ cally uniformly $B$-continuous on $[0, T] \times H$ if it is uniformly continuous in the $|\cdot| \times\|\cdot\|_{-1}$ norm on bounded subsets of $[0, T] \times H$.

We will write $u^{*}$ and $u_{*}$ to denote the upper and lower semicontinuous envelopes of $u$ in the $|\cdot| \times\|\cdot\|_{-1}$ norm, i.e.

$$
\begin{aligned}
& u^{*}(t, x)=\lim \sup \left\{u(s, y): s \rightarrow t,\|y-x\|_{-1} \rightarrow 0\right\}, \\
& u_{*}(t, x)=\lim \inf \left\{u(s, y): s \rightarrow t,\|y-x\|_{-1} \rightarrow 0\right\} .
\end{aligned}
$$

For a Hilbert space $V$ we will be using the following function spaces:

$$
\begin{aligned}
C^{2}(V) & =\left\{u: V \rightarrow \mathbb{R}: D u, D^{2} u \text { are continuous }\right\}, \\
C^{1,2}((0, T) \times V) & =\left\{u:(0, T) \times V \rightarrow \mathbb{R}: u_{t}, D u, D^{2} u \text { are continuous }\right\} .
\end{aligned}
$$

We will write $L(V)$ for the space of bounded, linear operators in $V$ equipped with the operator norm.

3. Viscosity solutions. In order to obtain Perron's method we will have to deal with discontinuous solutions. Therefore we need two definitions of viscosity solutions: the more usual one that is a stronger version of the definition from [25], and a discontinuous viscosity solution that is based on the notion given for first order equations by Ishii [17].

Definition 3.1. A function $\psi$ is a test function if $\psi=\varphi \pm \delta(t) h(\|x\|)$, where:

(i) $\varphi \in C^{1,2}((0, T) \times H), \varphi$ is $B$-continuous, and $\varphi_{t}, A^{*} D \varphi, D \varphi, D^{2} \varphi$ are uniformly continuous on closed subsets of $(0, T) \times H$. 
(ii) $\delta \in C^{1}((0, T))$ and $\delta>0$ on $(0, T)$.

(iii) $h \in C^{2}([0, \infty))$ and $h^{\prime}(0)=0, h^{\prime \prime}(0)>0, h^{\prime}(r)>0$ for $r \in(0, \infty)$.

For stationary equations $\varphi$ is independent of $t$ and $\delta(t) \equiv 1$.

We remark that even though $\|x\|$ is not differentiable at 0 , the function $h(\|x\|)$ is in $C^{2}(H)$ for a test function $h$ as above. Notice also that if $\|x\| \rightarrow 0$ then $h^{\prime}(\|x\|) /\|x\| \rightarrow h^{\prime \prime}(0)>0$ so the term $h^{\prime}(\|x\|) /\|x\|$ is bounded away from 0 on bounded sets.

Definition 3.2. A locally bounded $B$-upper semicontinuous function $u$ is a viscosity subsolution of (1.1) if whenever $u-\psi$ has a local maximum at a point $x$ for a test function $\psi=\varphi+h(\|x\|)$ then $x \in H_{1}$ and

$$
u(x)+\lambda\|x\|_{1}^{2} \frac{h^{\prime}(\|x\|)}{\|x\|}+\left\langle x, A^{*} D \varphi(x)\right\rangle+F\left(x, D \psi(x), D^{2} \psi(x)\right) \leq 0 .
$$

A locally bounded $B$-lower semicontinuous function $u$ is a viscosity supersolution of (1.1) if whenever $u-\psi$ has a local minimum at a point $x$ for a test function $\psi=\varphi-h(\|x\|)$ then $x \in H_{1}$ and

$$
u(x)-\lambda\|x\|_{1}^{2} \frac{h^{\prime}(\|x\|)}{\|x\|}+\left\langle x, A^{*} D \varphi(x)\right\rangle+F\left(x, D \psi(x), D^{2} \psi(x)\right) \geq 0 .
$$

A viscosity solution of (1.1) is a function which is both a viscosity subsolution and a viscosity supersolution.

Definition 3.3. A locally bounded $B$-upper semicontinuous function $u$ is a viscosity subsolution of (1.2) if whenever $u-\psi$ has a local maximum at a point $(t, x) \in(0, T) \times H$ for a test function $\psi(s, y)=\varphi(s, y)+\delta(s) h(\|y\|)$ then $x \in H_{1}$ and

$$
\begin{aligned}
\psi_{t}(t, x)+\lambda\|x\|_{1}^{2} \delta(t) \frac{h^{\prime}(\|x\|)}{\|x\|}+\langle x, & \left.A^{*} D \varphi(t, x)\right\rangle \\
& +F\left(t, x, D \psi(t, x), D^{2} \psi(t, x)\right) \leq 0 .
\end{aligned}
$$

A locally bounded $B$-lower semicontinuous function $u$ is a viscosity supersolution of (1.2) if whenever $u-\psi$ has a local minimum at a point $(t, x) \in(0, T) \times H$ for a test function $\psi(s, y)=\varphi(s, y)-\delta(s) h(\|y\|)$ then $x \in H_{1}$ and

$$
\begin{aligned}
\psi_{t}(t, x)-\lambda\|x\|_{1}^{2} \delta(t) \frac{h^{\prime}(\|x\|)}{\|x\|}+\left\langle x, A^{*} D \varphi(t, x)\right\rangle & \\
& +F\left(t, x, D \psi(t, x), D^{2} \psi(t, x)\right) \geq 0 .
\end{aligned}
$$

A viscosity solution of (1.2) is a function which is both a viscosity subsolution and a viscosity supersolution.

DEFINITION 3.4. A locally bounded function $u$ is a discontinuous viscosity subsolution of $(1.1)$ if whenever $(u-h(\|\cdot\|))^{*}-\varphi$ has a local maximum 
in the topology of $\|\cdot\|_{-1}$ at a point $x$ for test functions $\varphi, h(\|y\|)$ such that

$$
u(y)-h(\|y\|) \rightarrow-\infty \quad \text { as }\|y\| \rightarrow \infty
$$

then $x \in H_{1}$ and

$$
\begin{aligned}
(u-h(\|\cdot\|))^{*}(x)+h(\|x\|)+\lambda\|x\|_{1}^{2} \frac{h^{\prime}(\|x\|)}{\|x\|}+\left\langle x, A^{*} D \varphi(x)\right\rangle & \\
& +F\left(x, D \psi(x), D^{2} \psi(x)\right) \leq 0
\end{aligned}
$$

where $\psi=\varphi+h(\|y\|)$.

A locally bounded function $u$ is a discontinuous viscosity supersolution of (1.1) if whenever $(u+h(\|\cdot\|))_{*}-\varphi$ has a local minimum in the topology of $\|\cdot\|_{-1}$ at a point $x$ for test functions $\varphi, h(\|y\|)$ such that

$$
u(y)+h(\|y\|) \rightarrow \infty \quad \text { as }\|y\| \rightarrow \infty
$$

then $x \in H_{1}$ and

$$
\begin{aligned}
(u+h(\|\cdot\|))_{*}(x)-h(\|x\|)-\lambda\|x\|_{1}^{2} \frac{h^{\prime}(\|x\|)}{\|x\|} & +\left\langle x, A^{*} D \varphi(x)\right\rangle \\
& +F\left(x, D \psi(x), D^{2} \psi(x)\right) \geq 0,
\end{aligned}
$$

where $\psi=\varphi-h(\|y\|)$.

A discontinuous viscosity solution of (1.1) is a function which is both a discontinuous viscosity subsolution and a discontinuous viscosity supersolution.

Definition 3.5. A locally bounded function $u$ is a discontinuous viscosity subsolution of $(1.2)$ if whenever $(u-\delta(\cdot) h(\|\cdot\|))^{*}-\varphi$ has a local maximum in the topology of $|\cdot| \times\|\cdot\|_{-1}$ at a point $(t, x)$ for test functions $\varphi, \delta(s) h(\|y\|)$ such that

$$
u(s, y)-\delta(s) h(\|y\|) \rightarrow-\infty \quad \text { as }\|y\| \rightarrow \infty \text { locally uniformly in } s
$$

then $x \in H_{1}$ and

$$
\begin{aligned}
\psi_{t}(t, x)+\lambda\|x\|_{1}^{2} \delta(t) \frac{h^{\prime}(\|x\|)}{\|x\|}+\langle x, & \left.A^{*} D \varphi(t, x)\right\rangle \\
& +F\left(t, x, D \psi(t, x), D^{2} \psi(t, x)\right) \leq 0,
\end{aligned}
$$

where $\psi(s, y)=\varphi(s, y)+\delta(s) h(\|y\|)$.

A locally bounded function $u$ is a discontinuous viscosity supersolution of (1.2) if whenever $(u+\delta(\cdot) h(\|\cdot\|))_{*}-\varphi$ has a local minimum in the topology of $|\cdot| \times\|\cdot\|_{-1}$ at a point $(t, x)$ for test functions $\varphi, \delta(s) h(\|y\|)$ such that

$$
u(s, y)+\delta(s) h(\|y\|) \rightarrow \infty \quad \text { as }\|y\| \rightarrow \infty \text { locally uniformly in } s
$$

then $x \in H_{1}$ and 


$$
\begin{aligned}
\psi_{t}(t, x)-\lambda\|x\|_{1}^{2} \delta(t) \frac{h^{\prime}(\|x\|)}{\|x\|}+\langle x, & \left.A^{*} D \varphi(t, x)\right\rangle \\
& +F\left(t, x, D \psi(t, x), D^{2} \psi(t, x)\right) \geq 0,
\end{aligned}
$$

where $\psi(s, y)=\varphi(s, y)-\delta(s) h(\|y\|)$.

A discontinuous viscosity solution of (1.2) is a function which is both a discontinuous viscosity subsolution and a discontinuous viscosity supersolution.

If a subsolution (respectively, supersolution) in Definition 3.4 or 3.5 is $B$ upper semicontinuous (respectively, $B$-lower semicontinuous) then it is easy to see that Definitions 3.4 and 3.5 reduce to Definitions 3.2 and 3.3 respectively, since if $u$ is $B$-upper semicontinuous (respectively, $B$-lower semicontinuous) then $(u-h(\|\cdot\|))^{*}(x)=u(x)-h(\|x\|)$ (respectively, $(u+h(\|\cdot\|))_{*}(x)=$ $u(x)+h(\|x\|))$.

Lemma 3.6. Without loss of generality the maxima and minima in Definitions 3.2 and 3.3 can be assumed to be global and strict in the $|\cdot| \times\|\cdot\|$ norm and the maxima and minima in Definitions 3.4 and 3.5 can be assumed to be global and strict in the $|\cdot| \times\|\cdot\|_{-1}$ norm. However, it is not clear if they can be strict in the $|\cdot| \times\|\cdot\|$ norm. Finally, without loss of generality we can always assume that the functions in Definitions 3.2 and 3.3 satisfy (3.1)-(3.4). Moreover we can also assume that the functions in Definitions 3.4 and 3.5 satisfy

$$
\begin{aligned}
& (u-\delta(\cdot) h(\|\cdot\|))^{*}(t, x)-\varphi(t, x) \rightarrow-\infty, \\
& (u+\delta(\cdot) h(\|\cdot\|))_{*}(t, x)-\varphi(t, x) \rightarrow \infty
\end{aligned}
$$

as $\|x\| \rightarrow \infty$ locally uniformly in $t$.

Proof. Let $u$ be a $B$-upper semicontinuous function and let

$$
(u-h-\varphi)(x) \geq(u-h-\varphi)(y) \quad \text { for } y \in B_{R}(x)
$$

for some $R>0$, i.e. $u-h-\varphi$ has a local maximum at $x$ for test functions $\varphi$ and $h$. We will show that there exist test functions $\widetilde{\varphi}$ and $\widetilde{h}$ such that $D \widetilde{\varphi}(x)=D \varphi(x), D^{2} \widetilde{\varphi}(x)=D^{2} \varphi(x), D \widetilde{h}(x)=D h(x), D^{2} \widetilde{h}(x)=D^{2} h(x)$, and $u-\widetilde{h}-\widetilde{\varphi}$ has a strict global maximum at $x$. Let $g \in C^{2}([0, \infty))$ be an increasing function such that

$$
1+r^{2}+\sup _{\|y\| \leq r}|u(y)| \leq g(r) .
$$

Let $g_{1} \in C^{2}([0, \infty))$ be a function such that

$$
g_{1}(r)= \begin{cases}0, & r \leq\|x\|+1, \\ \text { increasing, }, & \|x\|+1<r<\|x\|+2, \\ g(r), & r \geq\|x\|+2\end{cases}
$$


Let $\varphi_{1} \in C^{2}([0, \infty))$ be defined by

$$
\varphi_{1}(r)= \begin{cases}r^{4}, & r \leq 1, \\ \text { increasing, } & 1<r<2, \\ 2, & r \geq 2\end{cases}
$$

Now for $n \geq 1$ consider the function

$$
\Phi_{n}(y)=u(y)-\varphi(y)-n \varphi_{1}\left(\|x-y\|_{-1}\right)-h(\|y\|)-g_{1}(\|y\|) .
$$

Obviously we have

$$
\Phi_{n}(x)=u(x)-\varphi(x)-h(\|x\|) .
$$

Suppose that for every $n$ there exists $y_{n}$ such that $\Phi_{n}\left(y_{n}\right) \geq \Phi_{n}(x)$. Then we must have $\left\|x-y_{n}\right\|_{-1} \rightarrow 0$ as $n \rightarrow \infty$ and $\left\|y_{n}\right\| \leq C$, i.e. $y_{n} \rightarrow x$. Since $u$ is $B$-upper semicontinuous and $\varphi$ is $B$-continuous, and $h$ is strictly increasing, this implies that $\left\|y_{n}\right\| \rightarrow\|x\|$, and therefore $y_{n} \rightarrow x$ in $H$. But then $y_{n} \in B_{R}(x)$ for large $n$ and so we get

$$
\Phi_{n}\left(y_{n}\right)<u(y)-\varphi(y)-h(\|y\|) \leq u(x)-\varphi(x)-h(\|x\|),
$$

which is a contradiction. Therefore there must exist $n_{0}$ such that $\Phi_{n_{0}}(y)<$ $\Phi_{n_{0}}(x)$ for $y \neq x$. It then follows easily that $\Phi_{n_{0}+1}$ has a strict global maximum at $x$. Therefore the conclusion follows by setting $\widetilde{\varphi}(y)=\varphi(y)-$ $\left(n_{0}+1\right) \varphi_{1}\left(\|x-y\|_{-1}\right)$ and $\widetilde{h}(\|y\|)=h(\|y\|)-g_{1}(\|y\|)$.

The fact that the maxima and minima in Definition 3.4 can be assumed to be global and strict in the $\|\cdot\|_{-1}$ norm is obvious and the final statements about the convergences at $\infty$ follow from the construction of $\widetilde{\varphi}$ and $\widetilde{h}$.

REMARK 3.7. There are other possibilities for the choice of test functions that would give good theory. For instance one can replace the functions $\varphi$ in Definition 3.1 by the functions satisfying

- $\varphi \in C^{1,2}\left((0, T) \times H_{-1}\right)$, and $\varphi_{t}, D \varphi, D^{2} \varphi$ are uniformly continuous on closed subsets of $(0, T) \times H_{-1}$.

In this case one needs to assume that $B^{1 / 2} A^{*} B^{1 / 2}$ is bounded and that (2.2) is satisfied for all $x \in H_{1}$. (The term $\left\langle A^{*} x, x\right\rangle$ is now well defined for $x \in H_{1}$.) Notice that if $B^{1 / 2} A^{*} B^{1 / 2}$ is bounded and $\psi=\varphi \pm \delta(s) h(\|y\|)$ then $\left\langle x, A^{*} D \psi(x)\right\rangle$ is well defined for $x \in H_{1}$ and so Definitions $3.2-3.5$ can be simplified by replacing the terms

$$
\pm \lambda\|x\|_{1}^{2} \delta(t) \frac{h^{\prime}(\|x\|)}{\|x\|}+\left\langle x, A^{*} D \varphi(t, x)\right\rangle
$$

wherever they appear in Definitions $3.2-3.5$ by a single term $\left\langle x, A^{*} D \psi(t, x)\right\rangle$.

REMARK 3.8. It follows from the proof of Lemma 3.6 that if we know a priori that $u$ has a certain growth at $\infty$ then we can obtain the same growth (at least quadratic) for $\widetilde{h}$. For instance, if $u$ has a polynomial growth at 
$\infty$ we can have $\widetilde{h}$ which is a polynomial of some special form for large $\|x\|$. This is important in applications to stochastic optimal control where we may want to impose additional conditions on test functions to be able to apply stochastic calculus. In these applications it may also be useful to assume that $h^{\prime}(r) / r$ is globally bounded away from 0 for the radial test functions $h$. To avoid technical difficulties it may then be more convenient to choose $h$ belonging to one particular class of functions, say certain polynomials with growth depending on the growth of sub- and supersolutions we are dealing with. All results presented in this paper would hold with an appropriate version of such a definition. This approach has been successfully employed in $[2,13-16]$. However, for such narrow classes of radial test functions the global and local definitions of viscosity solutions may no longer be equivalent.

4. Comparison principles. In this section we prove comparison principles for discontinuous viscosity solutions. We begin with the comparison result for the stationary case.

Theorem 4.1. Let (2.1) and (2.2) hold and let $F$ satisfy $(\mathbf{1})_{0},(\mathbf{2}),(\mathbf{3})$. Let $u$ be a viscosity subsolution and $v$ be a viscosity supersolution of (1.1) in the sense of Definition 3.4, and let $u,-v$ be bounded from above. Then

$$
\lim _{R \uparrow \infty} \lim _{r \downarrow 0} \sup \left\{u(x)-v(y):\|x-y\|_{-1} \leq r, x, y \in B_{R}\right\} \leq 0 .
$$

In particular $u \leq v$.

REMARK 4.2. Theorem 4.1 shows that a bounded viscosity solution of (1.1) in the sense of Definition 3.4 is uniformly continuous in $H_{-1}$ on bounded subsets of $H$ and therefore is a viscosity solution in the sense of Definition 3.2.

Proof of Theorem 4.1. We argue by contradiction. Assume that (4.1) does not hold. Then there exists a positive real number $\eta$ such that

$$
\lim _{R \uparrow \infty} \lim _{r \downarrow 0} \sup \left\{u(x)-v(y):\|x-y\|_{-1} \leq r, x, y \in B_{R}\right\}>2 \eta .
$$

Let $\mu(x)$ be the function satisfying $(\mathbf{1})_{0}$. For every positive real number $\alpha$, we define $u_{\alpha}(x)=u(x)-\alpha \mu(x)$ and $v_{\alpha}(y)=v(y)-\alpha \mu(y)$. Set

$$
\begin{aligned}
m & :=\lim _{R \uparrow \infty} \lim _{r \downarrow 0} \sup \left\{u(x)-v(y):\|x-y\|_{-1}<r, x, y \in B_{R}\right\}, \\
m_{\alpha} & :=\lim _{r \downarrow 0} \sup \left\{\left(u_{\alpha}\right)^{*}(x)-\left(v_{\alpha}\right)_{*}(y):\|x-y\|_{-1}<r\right\} \\
& =\lim _{r \downarrow 0} \sup \left\{u_{\alpha}(x)-v_{\alpha}(y):\|x-y\|_{-1}<r\right\} \\
m_{\alpha, \varepsilon} & :=\sup \left\{\left(u_{\alpha}\right)^{*}(x)-\left(v_{\alpha}\right)_{*}(y)-\frac{1}{2 \varepsilon}\|x-y\|_{-1}^{2}\right\},
\end{aligned}
$$


We have

$$
\begin{aligned}
m & =\lim _{\alpha \downarrow 0} m_{\alpha}, \\
m_{\alpha} & =\lim _{\varepsilon \downarrow 0} m_{\alpha, \varepsilon} .
\end{aligned}
$$

Using perturbed optimization techniques [24] (see also [6]) we obtain sequences $p_{n}, q_{n} \in H$ such that $\left\|p_{n}\right\|+\left\|q_{n}\right\| \rightarrow 0$ as $n \rightarrow \infty$, and

$$
\left(u_{\alpha}\right)^{*}(x)-\left(v_{\alpha}\right)_{*}(y)-\frac{1}{2 \varepsilon}\|x-y\|_{-1}^{2}+\left\langle B p_{n}, x\right\rangle+\left\langle B q_{n}, y\right\rangle
$$

achieves a strict global maximum at some point $(\bar{x}, \bar{y}) \in H \times H$. Convergences (4.2)-(4.3) yield

$$
\begin{gathered}
\lim _{\varepsilon \downarrow 0} \limsup _{n \rightarrow \infty} \frac{1}{2 \varepsilon}\|\bar{x}-\bar{y}\|_{-1}^{2}=0 \quad \text { for every } \alpha>0, \\
\quad \lim _{\alpha \downarrow 0} \limsup _{\varepsilon \downarrow 0} \limsup _{n \rightarrow \infty}(\alpha \mu(\bar{x})+\alpha \mu(\bar{y}))=0 .
\end{gathered}
$$

We now have

$$
\|x-y\|_{-1}^{2}=\left\|P_{N}(x-y)\right\|_{-1}^{2}+\left\|Q_{N}(x-y)\right\|_{-1}^{2}
$$

and

$$
\begin{aligned}
\left\|Q_{N}(x-y)\right\|_{-1}^{2} \leq & 2\left\langle B Q_{N}(\bar{x}-\bar{y}), x-y\right\rangle+2\left\|Q_{N}(x-\bar{x})\right\|_{-1}^{2} \\
& +2\left\|Q_{N}(y-\bar{y})\right\|_{-1}^{2}-\left\|Q_{N}(\bar{x}-\bar{y})\right\|_{-1}^{2}
\end{aligned}
$$

with equality at $\bar{x}, \bar{y}$. Therefore defining

$$
\begin{aligned}
u_{1}(x)= & \left(u_{\alpha}\right)^{*}(x)-\left\langle B Q_{N}(\bar{x}-\bar{y}), x\right\rangle / \varepsilon-\left\|Q_{N}(x-\bar{x})\right\|_{-1}^{2} / \varepsilon \\
& +\left\|Q_{N}(\bar{x}-\bar{y})\right\|_{-1}^{2} / 2 \varepsilon+\left\langle B p_{n}, x\right\rangle
\end{aligned}
$$

and

$$
v_{1}(y)=\left(v_{\alpha}\right)_{*}(y)-\left\langle B Q_{N}(\bar{x}-\bar{y}), y\right\rangle / \varepsilon+\left\|Q_{N}(y-\bar{y})\right\|_{-1}^{2} / \varepsilon-\left\langle B q_{n}, y\right\rangle,
$$

we see that

$$
u_{1}(x)-v_{1}(y)-\frac{1}{2 \varepsilon}\left\|P_{N}(x-y)\right\|_{-1}^{2}
$$

has a strict global maximum in $H_{-1}$ at $(\bar{x}, \bar{y})$. At this step we need to produce appropriate test functions to be able to use the definition of solution. This is done using partial sup-convolution techniques first introduced in [20] (see also [4]).

LEMMA 4.3. Given $N \geq 1$ there exist functions $\varphi_{k}, \psi_{k} \in C^{2}\left(H_{-1}\right)$ with uniformly continuous derivatives such that $u_{1}(x)-\varphi_{k}(x)$ has a global maximum at some point $x_{k}, v_{1}(y)+\psi_{k}(y)$ has a global minimum at some point $y_{k}$, 
and

$$
\begin{gathered}
\left(x_{k}, u_{1}\left(x_{k}\right), D \varphi_{k}\left(x_{k}\right), D^{2} \varphi_{k}\left(x_{k}\right)\right) \rightarrow\left(\bar{x}, u_{1}(\bar{x}), B P_{N}(\bar{x}-\bar{y}) / \varepsilon, X_{N}\right), \\
\left(y_{k}, v_{1}\left(y_{k}\right), D \psi_{k}\left(y_{k}\right), D^{2} \psi_{k}\left(y_{k}\right)\right) \rightarrow\left(\bar{y}, v_{1}(\bar{y}), B P_{N}(\bar{y}-\bar{x}) / \varepsilon, Y_{N}\right), \\
\left(\begin{array}{cc}
X_{N} & 0 \\
0 & Y_{N}
\end{array}\right) \leq \frac{2}{\varepsilon}\left(\begin{array}{cc}
B & -B \\
-B & B
\end{array}\right)
\end{gathered}
$$

with the convergences being in $H \times \mathbb{R} \times H_{2} \times L(H)$.

Proof. Set $x_{N}=P_{N} x, x_{N}^{\perp}=Q_{N} x, y_{N}=P_{N} y, y_{N}^{\perp}=Q_{N} y$ and define

$$
\widetilde{u}_{1}\left(x_{N}\right)=\sup _{x_{N}^{\perp} \in Q_{N} H} u_{1}\left(x_{N}+x_{N}^{\perp}\right), \quad \widetilde{v}_{1}\left(y_{N}\right)=\inf _{y_{N}^{\perp} \in Q_{N} H} v_{1}\left(y_{N}+y_{N}^{\perp}\right),
$$

the partial sup- and inf-convolutions of $u_{1}$ and $v_{1}$ respectively. Then

$$
\left(\widetilde{u}_{1}\right)^{*}\left(x_{N}\right)-\left(\widetilde{v}_{1}\right)_{*}\left(y_{N}\right)-\frac{1}{2 \varepsilon}\left\|x_{N}-y_{N}\right\|_{-1}^{2}
$$

has a strict global maximum over $V_{N} \times V_{N}$ at $\left(\bar{x}_{N}, \bar{y}_{N}\right)$, where $\left(\widetilde{u}_{1}\right)^{*}$ and $\left(\widetilde{v}_{1}\right)_{*}$ are the upper and lower semicontinuous envelopes of $\widetilde{u}_{1}$ and $\widetilde{v}_{1}$ in $V_{N}$. Moreover we have $\left(\widetilde{u}_{1}\right)^{*}\left(\bar{x}_{N}\right)=u_{1}(\bar{x})$ and $\left(\widetilde{v}_{1}\right)_{*}\left(\bar{y}_{N}\right)=v_{1}(\bar{y})$.

We can now apply the finite-dimensional maximum principle when we consider $V_{N}$ as a space with the topology inherited from $H_{-1}$ (which is equivalent to the topology inherited from $H$ ). Denote $V_{N}$ with this topology by $\widetilde{V}_{N}$. Therefore there exist bounded functions $\widetilde{\varphi}_{k}, \widetilde{\psi}_{k} \in C^{2}\left(\widetilde{V}_{N}\right)$ with uniformly continuous derivatives such that $\left(\widetilde{u}_{1}\right)^{*}\left(x_{N}\right)-\widetilde{\varphi}_{k}\left(x_{N}\right)$ has a strict global maximum at some point $\left(x_{N}^{k}\right),\left(\widetilde{v}_{1}\right)_{*}\left(y_{N}\right)+\widetilde{\psi}_{k}\left(y_{N}\right)$ has a strict global minimum at some point $\left(y_{N}^{k}\right)$, and as $k \rightarrow \infty$,

$$
\begin{aligned}
& \left(x_{N}^{k},\left(\widetilde{u}_{1}\right)^{*}\left(x_{N}^{k}\right), D_{\widetilde{V}_{N}} \widetilde{\varphi}_{k}\left(x_{N}^{k}\right), D_{\widetilde{V}_{N}}^{2} \widetilde{\varphi}_{k}\left(x_{N}^{k}\right)\right) \\
& \rightarrow\left(\bar{x}_{N}, u_{1}(\bar{x}),\left(\bar{x}_{N}-\bar{y}_{N}\right) / \varepsilon, \widetilde{X}_{N}\right), \\
& \left(y_{N}^{k},\left(\widetilde{v}_{1}\right)_{*}\left(y_{N}^{k}\right), D_{\widetilde{V}_{N}} \widetilde{\psi}_{k}\left(y_{N}^{k}\right), D_{\widetilde{V}_{N}}^{2} \widetilde{\psi}_{k}\left(y_{N}^{k}\right)\right) \\
& \rightarrow\left(\bar{y}_{N}, v_{1}(\bar{y}),\left(\bar{y}_{N}-\bar{x}_{N}\right) / \varepsilon, \widetilde{Y}_{N}\right), \\
& \left(\begin{array}{cc}
\widetilde{X}_{N} & 0 \\
0 & \widetilde{Y}_{N}
\end{array}\right) \leq \frac{2}{\varepsilon}\left(\begin{array}{cc}
I & -I \\
-I & I
\end{array}\right) \quad \text { in } H_{-1} \times H_{-1}
\end{aligned}
$$

for some $N \times N$ matrices $\widetilde{X}_{N}$ and $\widetilde{Y}_{N}$ that as operators in $L\left(H_{-1}\right)$ satisfy $X_{N}=P_{N} X_{N} P_{N}, Y_{N}=P_{N} Y_{N} P_{N}$ and are symmetric. Furthermore we can set the above maximum and minimum to be equal to zero. (Above, the symbols $D_{\widetilde{V}_{N}}$ and $D_{\widetilde{V}_{N}}^{2}$ denote the Fréchet derivatives in $\widetilde{V}_{N}$.) Since in $V_{N}$ the topology of $H_{-1}$ is equivalent to the topology of $H$ the above convergences hold in the topology of $H \times \mathbb{R} \times H \times \mathcal{S}(H)$. We now extend $\widetilde{\varphi}_{k}, \widetilde{\psi}_{k}$ to functions in $C^{2}\left(H_{-1}\right)$ by setting $\varphi_{k}(x)=\widetilde{\varphi}_{k}\left(P_{N} x\right)$ and $\psi_{k}(y)=\widetilde{\psi}_{k}\left(P_{N} y\right)$. Then, if 
$D_{H_{-1}} \varphi_{k}$ denotes the Fréchet derivative of $\varphi_{k}$ in $H_{-1}$ we have

$$
D \varphi_{k}(x)=B D_{H_{-1}} \varphi_{k}(x), \quad D^{2} \varphi_{k}(x)=B D_{H_{-1}}^{2} \varphi_{k}(x),
$$

and the same also holds for $\psi_{k}$.

Using a perturbed optimization result, we can find sequences $\widetilde{p}_{j}, \widetilde{q}_{j} \in H$ such that $\left\|\widetilde{p}_{j}\right\|+\left\|\widetilde{q}_{j}\right\| \rightarrow 0$ as $j \rightarrow \infty$, and

$$
\begin{gathered}
u_{1}(x)-\varphi_{k}(x)-\left\langle B \widetilde{p}_{j}, x\right\rangle \text { has a global maximum at some } x_{j} \\
v_{1}(y)+\psi_{k}(y)-\left\langle B \widetilde{q}_{j}, y\right\rangle \text { has a global minimum at some } y_{j}
\end{gathered}
$$

Combining (4.14) and the fact that $\left(\widetilde{u}_{1}\right)^{*}\left(x_{N}\right)-\widetilde{\varphi}_{k}\left(x_{N}\right)$ has a strict global maximum at $x_{N}^{k}$, we deduce that $P_{N} x_{j} \rightarrow x_{N}^{k}$ and $u_{1}\left(P_{N} x_{j}\right) \rightarrow\left(\widetilde{u}_{1}\right)^{*}\left(x_{N}^{k}\right)$ as $j \rightarrow \infty$. Similarly (4.15) and the fact that $\left(\widetilde{v}_{1}\right)_{*}\left(y_{N}\right)+\widetilde{\psi}_{k}\left(y_{N}\right)$ has a strict global minimum at some point $y_{N}^{k}$ implies that $P_{N} y_{j} \rightarrow y_{N}^{k}$ and $v_{1}\left(P_{N} y_{j}\right) \rightarrow\left(\widetilde{v}_{1}\right)^{*}\left(y_{N}^{k}\right)$ as $j \rightarrow \infty$. We can then select a subsequence $j_{k}$ such that $P_{N} x_{j_{k}} \rightarrow P_{N} \bar{x}$ and $P_{N} y_{j_{k}} \rightarrow P_{N} \bar{y}$, with the additional requirements that $u_{1}\left(P_{N} x_{j_{k}}\right) \rightarrow u_{1}(\bar{x}), v_{1}\left(P_{N} y_{j_{k}}\right) \rightarrow v_{1}(\bar{y}), \varphi_{k}\left(P_{N} x_{j_{k}}\right)-\varphi_{k}\left(\bar{x}_{N}\right) \rightarrow 0$ and $\psi_{k}\left(P_{N} y_{j_{k}}\right)-\psi_{k}\left(\bar{y}_{N}\right) \rightarrow 0$ as $k \rightarrow \infty$. Moreover we can choose the subsequence $j_{k}$ so that all the convergences in (4.10) hold when $x_{N}^{k}$ and $y_{N}^{k}$ are replaced by $P_{N} x_{j_{k}}$ and $P_{N} y_{j_{k}}$ respectively. We may now repeat rather standard arguments of [4] (see also [13, pp. 409-410]) and use (4.14) and (4.15) to show that $x_{j_{k}} \rightarrow \bar{x}$ and $y_{j_{k}} \rightarrow \bar{y}$ in $H_{-1}$.

We now need to prove that $x_{j_{k}} \rightarrow \bar{x}$ and $y_{j_{k}} \rightarrow \bar{y}$ in $H$. To obtain these convergences, it will be enough to prove that $x_{j_{k}}$ and $y_{j_{k}}$ are uniformly bounded in $H_{1}$ (independently of $k$ ). First we observe that $x_{j_{k}}$ and $y_{j_{k}}$ are uniformly bounded in $H$ (they remain in a ball whose radius depends exclusively on $\alpha$ ). Using (4.14) and the definition of subsolution we get

$$
\begin{aligned}
& \left(u_{\alpha}\right)^{*}\left(x_{j_{k}}\right)+2 \alpha \delta\left\|x_{j_{k}}\right\|_{1}^{2}+\alpha \mu\left(x_{j_{k}}\right) \\
& +\left\langle x_{j_{k}}, A^{*} B\left(-p_{n}+\widetilde{p}_{j_{k}}+Q_{N}(\bar{x}-\bar{y}) / \varepsilon+Q_{N}\left(\bar{x}-x_{j_{k}}\right) / \varepsilon\right)+A^{*} D \varphi_{k}\left(x_{j_{k}}\right)\right\rangle \\
& +F\left(x_{j_{k}},-B p_{n}+B \widetilde{p}_{j_{k}}+B Q_{N}(\bar{x}-\bar{y}) / \varepsilon+B Q_{N}\left(\bar{x}-x_{j_{k}}\right) / \varepsilon+D \varphi_{k}\left(x_{j_{k}}\right)\right. \\
& \left.+\alpha D \mu\left(x_{j_{k}}\right), B Q_{N} / \varepsilon+D^{2} \varphi_{k}\left(x_{j_{k}}\right)+D^{2} \mu\left(x_{j_{k}}\right)\right) \leq 0 .
\end{aligned}
$$

Note that $\left(u_{\alpha}\right)^{*}\left(x_{j_{k}}\right) \rightarrow\left(u_{\alpha}\right)^{*}(\bar{x})$ so using the local boundedness of $F$ we can deduce from the above inequality that $\left\|x_{j_{k}}\right\|_{1} \leq C$ for some positive constant $C$ independent of $k$. A similar argument can be used to prove that $y_{j_{k}}$ is bounded in $H_{1}$. Therefore $B^{1 / 2} x_{j_{k}} \rightarrow B^{1 / 2} \bar{x}, B^{-1 / 2} x_{j_{k}} \rightarrow B^{-1 / 2} \bar{x}$ and so

$$
\left\|x_{j_{k}}-\bar{x}\right\|^{2}=\left\langle B^{-1 / 2}\left(x_{j_{k}}-\bar{x}\right), B^{-1 / 2}\left(x_{j_{k}}-\bar{x}\right)\right\rangle \rightarrow 0
$$

as $k \rightarrow \infty$. The same argument also shows that $y_{j_{k}} \rightarrow \bar{y}$. Therefore the lemma holds with $\varphi_{k}(x):=\varphi_{k}(x)+\left\langle B \widetilde{p}_{j_{k}}, x\right\rangle$ and $\psi_{k}(y):=\psi_{k}(y)-\left\langle B \widetilde{q}_{j_{k}}, y\right\rangle$. 
We now finish the proof of the theorem. Using (4.14), Definition 3.4 and taking $\liminf$ as $k \rightarrow \infty$ we have

$$
\begin{aligned}
& \left(u_{\alpha}\right)^{*}(\bar{x})+\alpha \mu(\bar{x})+2 \alpha \lambda\|\bar{x}\|_{1}^{2}+\left\langle\bar{x}, A^{*} B(\bar{x}-\bar{y}) / \varepsilon\right\rangle-\left\langle\bar{x}, A^{*} B p_{n}\right\rangle \\
& \quad+F\left(\bar{x}, \alpha D \mu(x)-B p_{n}+B(\bar{x}-\bar{y}) / \varepsilon, \alpha D^{2} \mu(\bar{x})+X_{N}+B Q_{N} / \varepsilon\right) \leq 0 .
\end{aligned}
$$

Similarly (4.15) and Definition 3.4 yield

$$
\begin{aligned}
& \left(v_{\alpha}\right)_{*}(\bar{y})-\alpha \mu(\bar{y})-2 \lambda \alpha\|\bar{y}\|_{1}^{2}+\left\langle\bar{y}, A^{*} B(\bar{x}-\bar{y}) / \varepsilon\right\rangle+\left\langle\bar{y}, A^{*} B q_{n}\right\rangle \\
& \quad+F\left(\bar{y},-\alpha D \mu(y)+B q_{n}+B(\bar{x}-\bar{y}) / \varepsilon,-\alpha D^{2} \mu(\bar{y})-Y_{N}-B Q_{N} / \varepsilon\right) \geq 0 .
\end{aligned}
$$

We then use $(\mathbf{1})_{0}$ and $(\mathbf{2})$ to obtain

$$
\begin{aligned}
& \left(u_{\alpha}\right)^{*}(\bar{x})-\left(v_{\alpha}\right)_{*}(\bar{y}) \leq \sigma_{1}(N)+\sigma_{2}(n)+C_{0}\|\bar{x}-\bar{y}\|_{-1}^{2} / \varepsilon \\
& \quad+F\left(\bar{y}, B(\bar{x}-\bar{y}) / \varepsilon,-Y_{N}\right)-F\left(\bar{x}, B(\bar{x}-\bar{y}) / \varepsilon, X_{N}\right)+K \alpha(2+\mu(\bar{x})+\mu(\bar{y}))
\end{aligned}
$$

where $\sigma_{1}(N) \rightarrow 0$ for $n, \varepsilon, \alpha$ fixed and $\sigma_{2}(n) \rightarrow \infty$ for $\alpha, \varepsilon$ fixed. Finally, (3) gives

$$
\begin{aligned}
\left(u_{\alpha}\right)^{*}(\bar{x})- & \left(v_{\alpha}\right)_{*}(\bar{y}) \leq \sigma_{1}(N)+\sigma_{2}(n)+C_{0}\|\bar{x}-\bar{y}\|_{-1}^{2} / \varepsilon \\
& +\omega_{R(\alpha)}\left(\|x-y\|_{-1}\left(1+\|(x-y)\|_{-1} / \varepsilon\right)\right)+K \alpha(2+\mu(\bar{x})+\mu(\bar{y})) .
\end{aligned}
$$

If $m>2 \eta$, then for $\varepsilon, \alpha$ sufficiently small and $n$ large enough we have $\left(u_{\alpha}\right)^{*}(\bar{x})-\left(v_{\alpha}\right)_{*}(\bar{y})>\eta$. Therefore we get

$$
\begin{aligned}
\eta \leq & \sigma_{1}(N)+\sigma_{2}(n)+C_{0}\|\bar{x}-\bar{y}\|_{-1}^{2} / \varepsilon \\
& +\omega_{R(\alpha)}\left(\|x-y\|_{-1}\left(1+\|(x-y)\|_{-1} / \varepsilon\right)\right)+K \alpha(2+\mu(\bar{x})+\mu(\bar{y})) .
\end{aligned}
$$

Letting now $N \rightarrow \infty, n \rightarrow \infty, \varepsilon \rightarrow 0$ and $\alpha \rightarrow 0$ in that order we arrive at $\eta \leq 0$, which is a contradiction.

In the next theorem we prove a comparison result for the time dependent problem (1.2).

TheOrem 4.4. Let (2.1) and (2.2) hold and let $F$ satisfy $(\mathbf{1})_{k},(\mathbf{2}),(\mathbf{3})$ for some $k \geq 0$. Let $g$ be locally uniformly B-continuous and such that

$$
|g(x)| \leq C\left(1+\|x\|^{k}\right) \quad \text { for some } C>0 .
$$

Let $u$ be a viscosity subsolution of (1.2), and $v$ be a viscosity supersolution of (1.2) in the sense of Definition 3.5 such that

$$
\begin{gathered}
u,-v \leq C\left(1+\|x\|^{k}\right) \quad \text { for some } C>0, \\
\lim _{t \rightarrow 0}\left\{(u(t, x)-g(x))_{+}+(v(t, x)-g(x))_{-}\right\}=0
\end{gathered}
$$

uniformly on bounded sets. Then for every $0<T_{1}<T$,

$$
\begin{array}{r}
\lim _{R \uparrow \infty} \lim _{(r, \eta) \downarrow(0,0)} \sup \left\{u(t, x)-v(s, y):|t-s|<\eta,\|x-y\|_{-1}<r\right. \\
\left.x, y \in B_{R}, 0 \leq t, s \leq T_{1}\right\} \leq 0 .
\end{array}
$$

In particular $u \leq v$. 
Proof. We will just outline the proof as it is similar to the proof of Theorem 4.1. We argue by contradiction and assume that (4.20) is not true. Then for a sufficiently small $\sigma>0$ and some $\gamma>0$ we have

$$
\begin{gathered}
\gamma<\lim _{R \uparrow \infty} \lim _{(r, \eta) \downarrow(0,0)} \sup \left\{u^{\sigma}(t, x)-v^{\sigma}(s, y):\right. \\
\left.|t-s|<\eta,\|x-y\|_{-1}<r, x, y \in B_{R}\right\} \\
=\lim _{R \uparrow \infty} \lim _{r \downarrow 0} \lim _{\eta \downarrow 0} \sup \left\{u^{\sigma}(t, x)-v^{\sigma}(s, y):\right. \\
\left.|t-s|<\eta,\|x-y\|_{-1}<r, x, y \in B_{R}\right\},
\end{gathered}
$$

where we have set

$$
u^{\sigma}(t, x)=u(t, x)-\frac{\sigma}{T-t}, \quad v^{\sigma}(s, y)=v(s, y)+\frac{\sigma}{T-s} .
$$

We define

$$
u_{\alpha}(t, x)=u^{\sigma}(t, x)-\alpha e^{K t} \mu(x), \quad v_{\alpha}(s, y)=v^{\sigma}(s, y)+\alpha e^{K s} \mu(y),
$$

where $\mu(x)$ satisfies $(\mathbf{1})_{k}$ and $K$ is the constant from $(\mathbf{1})_{k}$. Let

$$
\begin{aligned}
m & :=\lim _{R \uparrow \infty} \lim _{r \downarrow 0} \lim _{\eta \downarrow 0} \sup \left\{u^{\sigma}(t, x)-v^{\sigma}(s, y):\right. \\
\left.\|x-y\|_{-1}<r,|t-s|<\eta, x, y \in B_{R}\right\}, & \\
m_{\alpha}: & =\lim _{r \downarrow 0} \lim _{\eta \downarrow 0} \sup \left\{\left(u_{\alpha}\right)^{*}(t, x)-\left(v_{\alpha}\right)_{*}(s, y):\|x-y\|_{-1}<r,|t-s|<\eta\right\} \\
= & \lim _{r \downarrow 0} \lim _{\eta \downarrow 0} \sup \left\{u_{\alpha}(t, x)-v_{\alpha}(s, y):\|x-y\|_{-1}<r,|t-s|<\eta\right\}, \\
m_{\alpha, \varepsilon}: & =\lim _{\eta \downarrow 0} \sup \left\{\left(u_{\alpha}\right)^{*}(t, x)-\left(v_{\alpha}\right)_{*}(s, y)-\frac{1}{2 \varepsilon}\|x-y\|_{-1}^{2}:|t-s|<\eta\right\}, \\
m_{\alpha, \varepsilon, \beta} & :=\sup \left\{\left(u_{\alpha}\right)^{*}(t, x)-\left(v_{\alpha}\right)_{*}(s, y)-\frac{1}{2 \varepsilon}\|x-y\|_{-1}^{2}-\frac{(t-s)^{2}}{2 \beta}\right\} .
\end{aligned}
$$

We have

$$
\begin{aligned}
m & =\lim _{\alpha \downarrow 0} m_{\alpha}, \\
m_{\alpha} & =\lim _{\varepsilon \downarrow 0} m_{\alpha, \varepsilon}, \\
m_{\alpha, \varepsilon} & =\lim _{\beta \downarrow 0} m_{\alpha, \varepsilon, \beta},
\end{aligned}
$$

however $m$ can now be $\infty$. Using perturbed optimization results, we can find sequences $a_{n}, b_{n} \in \mathbb{R}$ and $p_{n}, q_{n} \in H$ such that $\left|a_{n}\right|+\left|b_{n}\right|+\left\|p_{n}\right\|+\left\|q_{n}\right\| \leq 1 / n$ and

$$
\begin{aligned}
\left(u_{\alpha}\right)^{*}(t, x)-\left(v_{\alpha}\right)_{*}(s, y)-\frac{1}{2 \varepsilon}\|x-y\|_{-1}^{2}-\frac{1}{2 \beta}|t-s|^{2} \\
+a_{n} t+b_{n} s+\left\langle B p_{n}, x\right\rangle+\left\langle B q_{n}, y\right\rangle
\end{aligned}
$$

achieves a strict global maximum at some point $(\bar{t}, \bar{s}, \bar{x}, \bar{y}) \in(0, T] \times[0, T] \times$ $H \times H$. Convergences (4.22)-(4.24) yield 


$$
\lim _{\beta \downarrow 0} \limsup _{n \rightarrow \infty} \frac{1}{2 \beta}|\bar{t}-\bar{s}|^{2}=0 \quad \text { for every } \alpha, \varepsilon>0,
$$

(4.26) $\lim _{\varepsilon \downarrow 0} \limsup _{\beta \downarrow 0} \limsup _{n \rightarrow \infty} \frac{1}{2 \varepsilon}\|\bar{x}-\bar{y}\|_{-1}^{2}=0 \quad$ for every $\alpha>0$.

By the definition of $u^{\sigma}$ and $v^{\sigma}$ we have $\bar{t}<T$ and $\bar{s}<T$. In light of (4.19), (4.21)-(4.26), and the uniform continuity of $g$ in $H_{-1}$ on bounded sets of $H$, we can conclude that $\bar{t}>0$ and $\bar{s}>0$ for large $n$ and small $\beta, \varepsilon$. We define as before

$$
\begin{aligned}
u_{1}(t, x)= & \left(u_{\alpha}\right)^{*}(t, x)-\left\langle B Q_{N}(\bar{x}-\bar{y}), x\right\rangle / \varepsilon-\left\|Q_{N}(x-\bar{x})\right\|_{-1}^{2} / \varepsilon \\
& +\left\|Q_{N}(\bar{x}-\bar{y})\right\|_{-1}^{2} / 2 \varepsilon+a_{n} t+\left\langle B p_{n}, x\right\rangle
\end{aligned}
$$

and

$$
\begin{aligned}
v_{1}(s, y)= & \left(v_{\alpha}\right)_{*}(s, y)-\left\langle B Q_{N}(\bar{x}-\bar{y}), y\right\rangle / \varepsilon+\left\|Q_{N}(y-\bar{y})\right\|_{-1}^{2} / \varepsilon \\
& +\alpha \mu(y)-b_{n} s-\left\langle B q_{n}, y\right\rangle,
\end{aligned}
$$

so that

$$
u_{1}(t, x)-v_{1}(s, y)-\frac{1}{2 \varepsilon}\left\|P_{N}(x-y)\right\|_{-1}^{2}-\frac{1}{2 \beta}|t-s|^{2}
$$

has a strict global maximum at $(\bar{t}, \bar{s}, \bar{x}, \bar{y})$. Arguing now as in the proof of Lemma 4.3 we can claim the existence of functions $\varphi_{k}, \psi_{k} \in C^{1,2}((0, T) \times$ $\left.H_{-1}\right)$ with uniformly continuous derivatives such that $u_{1}(t, x)-\varphi_{k}(t, x)$ has a global maximum at some point $\left(t_{k}, x_{k}\right), v_{1}(s, y)+\psi_{k}(s, y)$ has a global minimum at some point $\left(s_{k}, y_{k}\right)$, and

$$
\begin{gathered}
\left(t_{k}, x_{k}, u_{1}\left(t_{k}, x_{k}\right), \frac{\partial \varphi_{k}}{\partial t}\left(t_{k}, x_{k}\right), D \varphi_{k}\left(t_{k}, x_{k}\right), D^{2} \varphi_{k}\left(t_{k}, x_{k}\right)\right) \\
\rightarrow\left(\bar{t}, \bar{x}, u_{1}(\bar{t}, \bar{x}),(\bar{t}-\bar{s}) / \beta, B P_{N}(\bar{x}-\bar{y}) / \varepsilon, X_{N}\right), \\
\left(s_{k}, y_{k}, v_{1}\left(s_{k}, y_{k}\right), \frac{\partial \psi_{k}}{\partial t}\left(s_{k}, y_{k}\right), D \psi_{k}\left(s_{k}, y_{k}\right), D^{2} \psi_{k}\left(s_{k}, y_{k}\right)\right) \\
\rightarrow\left(\bar{s}, \bar{y}, v_{1}(\bar{s}, \bar{y}),(\bar{s}-\bar{t}) / \beta, B P_{N}(\bar{y}-\bar{x}) / \varepsilon, Y_{N}\right), \\
\left(\begin{array}{cc}
X_{N} & 0 \\
0 & Y_{N}
\end{array}\right) \leq \frac{2}{\varepsilon}\left(\begin{array}{cc}
B & -B \\
-B & B
\end{array}\right),
\end{gathered}
$$

with the convergences in $\mathbb{R} \times H \times \mathbb{R} \times \mathbb{R} \times H_{2} \times L(H)$.

Therefore, using Definition 3.5 we find as in the proof of Theorem 4.1 that

$$
\begin{array}{r}
\frac{\sigma}{(T-\bar{t})^{2}}-a_{n}+\frac{\bar{t}-\bar{s}}{\beta}+\alpha K e^{K \bar{t}} \mu(\bar{x})+\alpha e^{K \bar{t}} \lambda \frac{\mu^{\prime}(\|\bar{x}\|)}{\|\bar{x}\|}\|\bar{x}\|_{1}^{2} \\
+\left\langle\bar{x}, A^{*} B(\bar{x}-\bar{y}) / \varepsilon\right\rangle-\left\langle\bar{x}, A^{*} B p_{n}\right\rangle \\
+F\left(\bar{t}, \bar{x}, \alpha e^{K \bar{t}} D \mu(\bar{x})-B p_{n}+B(\bar{x}-\bar{y}) / \varepsilon, \alpha e^{K \bar{t}} D^{2} \mu(\bar{x})+X_{N}+B Q_{N} / \varepsilon\right) \\
\leq 0
\end{array}
$$


and

$$
\begin{array}{r}
(4.31) \begin{array}{r}
b_{n}-\frac{\sigma}{(T-\bar{s})^{2}}+\frac{\bar{t}-\bar{s}}{\beta}-\alpha K e^{K \bar{s}} \mu(\bar{y})-\alpha e^{K \bar{s}} \lambda \frac{\mu^{\prime}(\|\bar{y}\|)}{\|\bar{y}\|}\|\bar{y}\|_{1}^{2} \\
+\left\langle\bar{y}, A^{*} B(\bar{x}-\bar{y}) / \varepsilon\right\rangle+\left\langle\bar{y}, A^{*} B q_{n}\right\rangle \\
+F\left(\bar{s}, \bar{y},-\alpha e^{K \bar{s}} D \mu(\bar{y})+B q_{n}+B(\bar{x}-\bar{y}) / \varepsilon,-\alpha e^{K \bar{s}} D^{2} \mu(\bar{y})-Y_{N}-B Q_{N} / \varepsilon\right) \\
\geq 0
\end{array} \\
\geq 0
\end{array}
$$

Combining (4.30) and (4.31) and using assumptions $(\mathbf{1})_{k},(\mathbf{2})$, and the local uniform continuity of $F$, we have

$$
\begin{aligned}
2 \sigma / T^{2} \leq & \sigma_{1}(N)+\sigma_{2}(n)+C_{0}\|\bar{x}-\bar{y}\|_{-1}^{2} / \varepsilon-\alpha K\left(e^{K \bar{t}} \mu(\bar{x})+e^{K \bar{s}} \mu(\bar{y})\right) \\
& +F\left(\bar{s}, \bar{y}, B(\bar{x}-\bar{y}) / \varepsilon,-Y_{N}\right)-F\left(\bar{t}, \bar{x}, B(\bar{x}-\bar{y}) / \varepsilon, X_{N}\right) \\
& +\alpha K\left(2+e^{K \bar{t}} \mu(\bar{x})+e^{K \bar{s}} \mu(\bar{y})\right)
\end{aligned}
$$

where $\sigma_{1}(N) \rightarrow 0$ for $n, \varepsilon, \alpha, \beta$ fixed and $\sigma_{2}(n) \rightarrow \infty$ for $\alpha, \varepsilon, \beta$ fixed. Using (3) and the local uniform continuity of $F$ we then conclude that

$$
\begin{aligned}
\sigma / T^{2} \leq & \sigma_{1}(N)+\sigma_{2}(n)+\sigma_{3}(\beta)+C_{0}\|\bar{x}-\bar{y}\|_{-1}^{2} / \varepsilon \\
& +\omega_{R(\alpha)}\left(\|x-y\|_{-1}\left(1+\|x-y\|_{-1} / \varepsilon\right)\right)+2 \alpha K,
\end{aligned}
$$

where $\sigma_{3}(\beta) \rightarrow 0$ for $\alpha$, $\varepsilon$ fixed. We can now let $N \rightarrow \infty, n \rightarrow \infty, \beta \rightarrow 0$, $\varepsilon \rightarrow 0$, and $\alpha \rightarrow 0$ in this order to arrive at $\sigma \leq 0$, which is a contradiction.

5. Perron's method and existence of solutions. In this section we will show that viscosity solutions of (1.1) in the sense of Definition 3.4 (respectively, viscosity solutions of (1.2) in the sense of Definition 3.5) can be obtained by Perron's method, i.e. by taking the supremum of all such viscosity subsolutions of (1.1) (respectively, (1.2)) provided that a viscosity subsolution and a viscosity supersolution exist. Therefore, if the assumptions of Theorem 4.1 (respectively, Theorem 4.4) are satisfied, we will deduce that the solution produced by this method is $B$-continuous, and so it is a viscosity solution in the sense of Definition 3.2 (respectively, Definition 3.3). A posteriori this will also show that this solution is equal to the supremum of all viscosity subsolutions in the sense of Definition 3.2 (respectively, Definition $3.3)$.

It is convenient to state a simple lemma for future reference.

LEMMA 5.1. Let (2.2) hold. Let $\varphi \in C^{2}(H)$ be B-upper semicontinuous and such that $A^{*} D \varphi$ is continuous, and let $h \in C^{2}((-\infty, \infty))$ be even and such that $h^{\prime}(r) \geq 0$ for $r \in(0, \infty)$. Let $w(x)=\varphi(x)-h(\|x\|)$ (respectively, $w(x)=-\varphi(x)+h(\|x\|))$ satisfy

$$
w(x)+\left\langle x, A^{*} D w(x)\right\rangle+F\left(x, D w(x), D^{2} w(x)\right) \leq 0 \quad \text { for } x \in D\left(A^{*}\right)
$$

(respectively, 


$$
\left.w(x)+\left\langle x, A^{*} D w(x)\right\rangle+F\left(x, D w(x), D^{2} w(x)\right) \geq 0 \quad \text { for } x \in D\left(A^{*}\right) .\right)
$$

Then $w$ is a viscosity subsolution (respectively, supersolution) of (1.1).

Proof. We will only give the proof in the subsolution case. Since $w$ is $B$ upper semicontinuous Definitions 3.2 and 3.4 are equivalent. Suppose that $w(y)-h_{1}(\|y\|)-\varphi_{1}(y)$ has a local maximum at $x$ for test functions $\varphi_{1}, h_{1}$. Then

$$
D \varphi(x)-\frac{h^{\prime}(\|x\|)}{\|x\|} x-D \varphi_{1}(x)-\frac{h_{1}^{\prime}(\|x\|)}{\|x\|} x=0
$$

and

$$
D^{2} w(x) \leq D^{2}\left(\varphi_{1}+h(\|\cdot\|)\right)(x) .
$$

Therefore either $x=0$ or

$$
\left(\frac{h^{\prime}(\|x\|)}{\|x\|}+\frac{h_{1}^{\prime}(\|x\|)}{\|x\|}\right) x=D \varphi(x)-D \varphi_{1}(x) \in D\left(A^{*}\right) \subset H_{1} .
$$

Define $\psi=\varphi_{1}+h_{1}(\|y\|)$. Now, using (2.2) and the degenerate ellipticity of $F$, we obtain

$$
\begin{aligned}
& w(x)+\lambda\|x\|_{1}^{2} \frac{h_{1}^{\prime}(\|x\|)}{\|x\|}+\left\langle x, A^{*} D \varphi_{1}(x)\right\rangle+F\left(x, D \psi(x), D^{2} \psi(x)\right) \\
& \leq w(x)+\frac{h_{1}^{\prime}(\|x\|)}{\|x\|}\left\langle x, A^{*} x\right\rangle+\left\langle x, A^{*} D \varphi_{1}(x)\right\rangle+F\left(x, D w(x), D^{2} w(x)\right) \\
& =w(x)+\left\langle x, A^{*} D w(x)\right\rangle+F\left(x, D w(x), D^{2} w(x)\right) \leq 0
\end{aligned}
$$

and the claim is proved.

Proposition 5.2. Let (2.2) be satisfied. Let $\mathcal{A}$ be a family of viscosity subsolutions of (1.1) in the sense of Definition 3.4. Suppose that the function

$$
u(x)=\sup \{w(x): w \in \mathcal{A}\}
$$

is locally bounded. Then $u$ is a viscosity subsolution of (1.1) in the sense of Definition 3.4.

Proof. Suppose that $(u-h(\|\cdot\|))^{*}-\varphi$ has a strict (in the $\|\cdot\|_{-1}$ norm) global maximum at a point $x$ for test functions $h$ and $\varphi$. (We can assume that $(u(y)-h(\|\cdot\|))^{*}(y)-\varphi(y) \rightarrow-\infty$ as $\|y\| \rightarrow \infty$.) Perturbed optimization techniques and Definition 3.2 then show that there exist viscosity subsolutions $w_{n}$ of (1.1), $x_{n} \in H_{1}$ and $p_{n} \in H$ with $\left\|p_{n}\right\| \leq 1 / n$ such that

$$
B^{1 / 2} x_{n} \rightarrow B^{1 / 2} x, \quad x_{n} \rightarrow x \quad \text { in } H \text { as } n \rightarrow \infty,
$$

$\left(w_{n}(y)-h(\|y\|)\right)^{*}-\varphi(y)-\left\langle B p_{n}, y\right\rangle$ has a strict (in the $\|\cdot\|_{-1}$ norm) global maximum at $x_{n}$, and

$$
\left(w_{n}-h(\|\cdot\|)\right)^{*}\left(x_{n}\right) \rightarrow(u-h(\|\cdot\|))^{*}(x) \quad \text { as } n \rightarrow \infty .
$$


Therefore, setting $\psi(y)=\varphi(y)-h(\|y\|)$, we have

$$
\begin{gathered}
\left(w_{n}-h(\|\cdot\|)\right)^{*}\left(x_{n}\right)-h\left(\left\|x_{n}\right\|\right)+\lambda\left\|x_{n}\right\|_{1}^{2} \frac{h^{\prime}\left(\left\|x_{n}\right\|\right)}{\left\|x_{n}\right\|} \\
+\left\langle x_{n}, A^{*} D \varphi\left(x_{n}\right)\right\rangle+\left\langle x_{n}, A^{*} B p_{n}\right\rangle+F\left(x_{n}, D \psi\left(x_{n}\right)+B p_{n}, D^{2} \psi\left(x_{n}\right)\right) \leq 0 .
\end{gathered}
$$

Since the $x_{n}$ are bounded, using the local boundedness of $F$ we thus obtain $\left\|x_{n}\right\|_{1}^{2} \leq C$ for some constant $C$, which, together with (5.2), implies that $x \in H_{1}$, and $B^{-1 / 2} x_{n} \rightarrow B^{-1 / 2} x$ as $n \rightarrow \infty$. Therefore, by (5.2),

$$
\left\|x_{n}-x\right\|^{2}=\left\langle B^{-1 / 2}\left(x_{n}-x\right), B^{1 / 2}\left(x_{n}-x\right)\right\rangle \rightarrow 0 \quad \text { as } n \rightarrow \infty,
$$

i.e. $x_{n} \rightarrow x$ in $H$. Using this, (5.3), the continuity of $F$, and the fact that $\|\cdot\|_{1}$ is lower semicontinuous in $H$, we can now pass to $\lim \inf$ as $n \rightarrow \infty$ in (5.4) to obtain

$$
\begin{aligned}
(u-h(\|\cdot\|))^{*}(x)+h(\|x\|)+\lambda\|x\|_{1}^{2} \frac{h^{\prime}(\|x\|)}{\|x\|}+\left\langle x, A^{*} D \varphi(x)\right\rangle \\
+F\left(x, D \psi(x), D^{2} \psi(x)\right) \leq 0
\end{aligned}
$$

which completes the proof.

Proposition 5.3. Let (2.2) be satisfied. Let $u_{0}, v_{0}$ be respectively a viscosity subsolution and a viscosity supersolution of (1.1) in the sense of Definition 3.4 such that $u_{0} \leq v_{0}$. Then the function

$$
u(x)=\sup \left\{w(x): u_{0} \leq w \leq v_{0}, w\right. \text { is a viscosity subsolution }
$$

$$
\text { of (1.1) in the sense of Definition } 3.4\}
$$

is a viscosity solution of (1.1) in the sense of Definition 3.4.

Proof. The fact that $u$ is a subsolution follows from Proposition 5.2. Suppose now that $(u+h(\|\cdot\|))_{*}-\varphi$ has a strict (in the $\|\cdot\|_{-1}$ norm) global minimum at a point $x$ for test functions $h(\|y\|)$ and $\varphi$. First we notice that if

$$
(u+h(\|\cdot\|))_{*}(x)=\left(v_{0}+h(\|\cdot\|)\right)_{*}(x)
$$

then $\left(v_{0}+h(\|\cdot\|)\right)_{*}-\varphi$ has a global minimum at $x$ and so we are done since $v_{0}$ is a viscosity supersolution. Therefore we only need to consider the case

$$
(u+h(\|\cdot\|))_{*}(x)<\left(v_{0}+h(\|\cdot\|)\right)_{*}(x) .
$$

It then follows from the $B$-continuity of $\varphi$ and the weak lower semicontinuity of $\|\cdot\|$ that for every $R>0$,

$$
\begin{aligned}
\varepsilon+(u+h(\|\cdot\|))_{*}(x)-\varphi & (x)+\varphi(y)-h(\|y\|) \\
& <\left(v_{0}+h(\|\cdot\|)\right)_{*}(y)-h(\|y\|) \leq v_{0}(y)
\end{aligned}
$$

for $y \in B_{-1}(x, r) \cap B(x, R)$ for some small $r, \varepsilon>0$. Set

$$
w(y)=\varepsilon+(u+h(\|\cdot\|))_{*}(x)-\varphi(x)+\varphi(y)-h(\|y\|) .
$$


If the condition for $u$ being a viscosity supersolution of (1.1) is violated at $x$ then either

(i) $x \notin H_{1}$, or

(ii) $x \in H_{1}$ but

$$
\begin{aligned}
(u+h(\|\cdot\|))_{*}(x)-h(\|x\|)- & \lambda\|x\|_{1}^{2} \frac{h^{\prime}(\|x\|)}{\|x\|}+\left\langle x, A^{*} D \varphi(x)\right\rangle \\
& +F\left(x, D \psi(x), D^{2} \psi(x)\right)<-\nu<0,
\end{aligned}
$$

where $\psi(y)=\varphi(y)-h(\|y\|), \nu>0$.

If (i) happens, i.e. if $x \notin H_{1}$ then

$$
\liminf _{y \rightarrow x \text { in } H_{-1}, y \in H_{1}}\|y\|_{1}=\infty .
$$

Otherwise we would have a sequence $y_{n}$ such that $B^{1 / 2} y_{n} \rightarrow B^{1 / 2} x$ and $\left\|B^{-1 / 2} y_{n}\right\| \leq C$. Then for some subsequence (still denoted by $y_{n}$ ) $B^{-1 / 2} y_{n}$ $\rightarrow z$ for some $z \in H$. But this implies that $x \in H_{1}$ and $z=B^{-1 / 2} x$. By the local boundedness of $F$, condition (5.8) now implies that for every $R>0$,

$$
w(y)-\lambda\|y\|_{1}^{2} \frac{h^{\prime}(\|y\|)}{\|y\|}+\left\langle y, A^{*} D \varphi(y)\right\rangle+F\left(y, D w(y), D^{2} w(y)\right)<-\nu / 2
$$

for $y \in B_{-1}(x, r) \cap B(x, R) \cap H_{1}$ for some small $r>0$.

Suppose that (ii) is true. We will show that for every $R>0,(5.9)$ holds for $y \in B_{-1}(x, r) \cap B(x, R) \cap H_{1}$ for $\varepsilon=\mu / 4$ and some small $r>0$. If not there exists a sequence $x_{n} \rightarrow x$ in $H_{-1}$ with $\left\|x_{n}\right\| \leq R$ such that

$$
\begin{aligned}
w\left(x_{n}\right)-\lambda\left\|x_{n}\right\|_{1}^{2} \frac{h^{\prime}\left(\left\|x_{n}\right\|\right)}{\left\|x_{n}\right\|} & +\left\langle x_{n}, A^{*} D \varphi\left(x_{n}\right)\right\rangle \\
& +F\left(x_{n}, D w\left(x_{n}\right), D^{2} w\left(x_{n}\right)\right) \geq-\nu / 2 .
\end{aligned}
$$

Then of course $\left\|x_{n}\right\|_{1} \leq C$ for some constant $C$ as otherwise (5.10) would be violated. But then we must have $B^{-1 / 2} x_{n} \rightarrow B^{-1 / 2} x$ in $H$ and so arguing as in the proof of Proposition 5.2 we obtain $x_{n} \rightarrow x$. However, then (5.7) and the lower semicontinuity of $\|\cdot\|_{1}$ imply

$$
\begin{aligned}
\limsup _{n \rightarrow \infty}\left(w\left(x_{n}\right)-\lambda\left\|x_{n}\right\|_{1}^{2} \frac{h^{\prime}\left(\left\|x_{n}\right\|\right)}{\left\|x_{n}\right\|}\right. & +\left\langle x_{n}, A^{*} D \varphi\left(x_{n}\right)\right\rangle \\
& \left.+F\left(x_{n}, D w\left(x_{n}\right), D^{2} w\left(x_{n}\right)\right)\right)<-\frac{\nu}{2}
\end{aligned}
$$

which gives a contradiction.

So we have shown that in either case, for every $R>0,(5.9)$ is satisfied for $y \in B_{-1}(x, r) \cap B(x, R) \cap H_{1}$ for some small $r, \varepsilon>0$.

It follows from the fact that $(u+h(\|\cdot\|))_{*}-\varphi$ has a (strict in the $\|\cdot\|_{-1}$ norm) global minimum at $x$ that for every $r>0$ if $\varepsilon$ is small enough 
(depending on $r$ ) there exists a constant $\mu_{r}>0$ such that

$$
w(y)<(u+h(\|\cdot\|))_{*}(y)-h(\|y\|)-\mu_{r} \leq u(y)-\mu_{r} \quad \text { for } y \notin B_{-1}(x, r) .
$$

Moreover it is clear by the growth condition on the test function in Definition 3.4 that if $R$ is large enough then

$$
w(y) \leq u(y)-1 \quad \text { for } y \notin B(x, R) .
$$

Using the last two inequalities and (5.6) we conclude that there exist $R, r, \varepsilon, \mu$ $>0$ such that

$$
w \leq v_{0} \quad \text { in } H, \quad w(y)<u(y)-\mu \quad \text { for } y \notin B_{-1}(x, r) \cap B(x, R),
$$
and (5.9) is satisfied for $y \in B_{-1}(x, r) \cap B(x, R) \cap H_{1}$.

Finally, if $R$ is large enough there exist $y_{n} \in B(x, R)$ such that $y_{n} \rightarrow x$ in $H_{-1}$ such that

$$
u\left(y_{n}\right)+h\left(\left\|y_{n}\right\|\right)-\varphi\left(y_{n}\right) \rightarrow(u+h(\|\cdot\|))_{*}(x)-\varphi(x),
$$

which means that there exist points $y \in B_{-1}(x, r) \cap B(x, R)$ for which

$$
u(y)<w(y) .
$$

We now claim that the function $w$ is a viscosity subsolution of (1.1) in $B_{-1}(x, r) \cap B(x, R)$. (Since $w$ is $B$-upper semicontinuous Definitions 3.2 and 3.4 are equivalent, but we point out that being a viscosity subsolution of (1.1) in $B_{-1}(x, r) \cap B(x, R)$ still requires that the maxima in Definition 3.4 be local in the $\|\cdot\|_{-1}$ norm in the whole space.) The claim follows from Lemma 5.1 upon noticing that by (5.9) and (2.2) we have

$$
\begin{aligned}
& w(y)+\left\langle y, A^{*} D w(y)\right\rangle+F\left(y, D w(y), D^{2} w(y)\right) \\
& \leq w(y)-\lambda\|y\|_{1}^{2} \frac{h^{\prime}(\|y\|)}{\|y\|}+\left\langle y, A^{*} D \varphi(y)\right\rangle+F\left(y, D w(y), D^{2} w(y)\right)<0
\end{aligned}
$$

for $y \in B_{-1}(x, r) \cap B(x, R) \cap D\left(A^{*}\right)$.

It now remains to show that the function $u_{1}=\max (w, u)$ is a viscosity subsolution in the sense of Definition 3.4 and $u_{0} \leq u_{1} \leq v_{0}$. But this is clear from Proposition 5.2 (more precisely from its proof) and the fact that $w$ is a viscosity subsolution of $(1.1)$ in $B_{-1}(x, r) \cap B(x, R)$, and (5.11). This, together with (5.12), gives us a contradiction, and the proof is complete.

Combining the above proposition with Theorem 4.1 we obtain the following result.

Theorem 5.4. Let (2.1) and (2.2) hold and let $F$ satisfy $(\mathbf{1})_{0},(\mathbf{2}),(\mathbf{3})$. Let $u_{0}, v_{0}$ be respectively a bounded viscosity subsolution and a bounded viscosity supersolution of (1.1) in the sense of Definition 3.4. Then the function

$$
u(x)=\sup \left\{w(x): u_{0} \leq w \leq v_{0}, w\right. \text { is a viscosity subsolution }
$$

$$
\text { of (1.1) the sense of Definition 3.4\} }
$$


is the unique bounded viscosity solution of (1.1) in the sense of Definition 3.2. Moreover $u$ is locally uniformly B-continuous.

The same technique applies to the time dependent problems (1.2). We just state here the final existence result that can be proved in the same way as Theorem 5.4 .

THEOREM 5.5. Let (2.1) and (2.2) hold and let $F$ satisfy $(\mathbf{1})_{k},(\mathbf{2}),(\mathbf{3})$ for some $k \geq 0$. Let $g$ be locally uniformly B-continuous and such that

$$
|g(x)| \leq C\left(1+\|x\|^{k}\right) \quad \text { for some } C>0 \text {. }
$$

Let $u_{0}$ be a viscosity subsolution of (1.2), and $v_{0}$ be a viscosity supersolution of (1.2) in the sense of Definition 3.5 such that

$$
\begin{gathered}
u_{0},-v_{0} \leq C\left(1+\|x\|^{k}\right) \quad \text { for some } C>0, \\
\lim _{t \rightarrow 0}\left\{\left|u_{0}(t, x)-g(x)\right|+\left|v_{0}(t, x)-g(x)\right|\right\}=0
\end{gathered}
$$

uniformly on bounded sets. Then the function

$$
\begin{aligned}
u(t, x)=\sup \left\{w(t, x): u_{0} \leq w \leq\right. & v_{0}, w \text { is a viscosity subsolution } \\
& \text { of }(1.2) \text { the sense of Definition } 3.5\}
\end{aligned}
$$

is the unique viscosity solution of (1.2) in the sense of Definition 3.3 satisfying (5.14) and (5.15). Moreover $u$ is locally uniformly B-continuous on $\left[0, T_{1}\right] \times H$ for every $0<T_{1}<T$.

We will construct a subsolution and a supersolution so that we can apply Perron's method. We remark that if $\sup _{x \in H}|F(x, 0,0)|=M<\infty$, then the functions $u(x)=-M$ and $v(x)=M$ are respectively a viscosity subsolution and a viscosity supersolution of (1.1) in the sense of Definition 3.2. In the proposition below, we will show how the construction of the supersolution can be done in the time dependent case. The construction of a subsolution is very similar.

Proposition 5.6. Let (2.2) hold and let $g$ be locally uniformly B-continuous with $|g(x)| \leq \mu(1+\|x\|)$ for $x \in H$ for some positive constant $\mu$. Then there is a viscosity supersolution $V$ of equation (1.2) such that $\lim _{t \downarrow 0} V(t, x)$ $=g(x)$ uniformly on bounded sets of $H$.

Proof. Define $C(r)=\sup \{|F(t, x, p, X)|: x \in H, t \in[0, T],\|p\|,\|X\|$ $\leq r\}$. Let $v(t, x)=\alpha t+2 \mu \sqrt{1+\|x\|^{2}}$. By a time dependent version of Lemma 5.1 a condition for $v$ to be a viscosity supersolution of (1.2) is

$$
\alpha+F\left(t, x, D v(t, x), D^{2} v(t, x)\right) \geq 0
$$

for all $(t, x) \in(0, T) \times H$. Since $D v(t, x)$ and $D^{2} v(t, x)$ are bounded we can therefore select $\alpha$ depending only on $\mu$ such that the above condition is satisfied. 
Let $z \in H$ and $\varepsilon>0$. We first choose a constant $R=R(\|z\|)$ such that $(\|x\|-\|z\|)_{+}^{4} \geq v(t, x)$ for $\|x\| \geq R$. We then find $M=M(\|z\|, \varepsilon)$ such that

$$
\bar{w}_{z, \varepsilon}(x)=g(z)+\varepsilon+M\|x-z\|_{-1}^{2}+(\|x\|-\|z\|)_{+}^{4} \geq g(x)
$$

for $\|x\| \leq R$. Let now $\gamma=\sup \left\{\left\|D \bar{w}_{z, \varepsilon}(x)\right\|+\left\|D^{2} \bar{w}_{z, \varepsilon}(x)\right\|:\|x\| \leq R\right\}$. In order for $w_{z, \varepsilon}(t, x)=\beta t+\bar{w}_{z, \varepsilon}(x)$ to be a viscosity supersolution of (1.2) in $(0, T) \times B(0, R)$ we need

$$
\beta+\left\langle x, A^{*} B(x-z)\right\rangle+F\left(t, x, D w_{z, \varepsilon}(t, x), D^{2} w_{z, \varepsilon}(t, x)\right) \geq 0 .
$$

This can be achieved by taking $\beta=R(R+\|z\|)\left\|A^{*} B\right\|+C(\gamma)$.

It now follows that

$$
\widehat{\omega}_{z, \varepsilon}(t, x)=\min \left\{w_{z, \varepsilon}(t, x), v(t, x)\right\}
$$

is a $B$-lower semicontinuous viscosity supersolution of $(1.2)$ in $(0, T) \times H$. It is clear from the construction of the $\widehat{\omega}_{z, \varepsilon}$ and the time dependent version of Proposition 5.2 for supersolutions that the function $V(t, x)=\inf _{z, \varepsilon} \widehat{\omega}_{z, \varepsilon}(t, x)$ is a viscosity supersolution of (1.2) in the sense of Definition 3.5 such that $\lim _{t \downarrow 0} V(t, x)=g(x)$ uniformly on bounded subsets of $H$.

6. Relaxed limits. In this section we show how the method of halfrelaxed limits of Barles-Perthame can be generalized to infinite-dimensional spaces. We consider two separate cases. The first deals with limits of suband supersolutions of equations on the whole space with operators $A$ satisfying similar structure conditions. The second deals with limits of finitedimensional approximations.

Let $F_{n}:[0, T] \times H \times H \times \mathcal{S}(H) \rightarrow \mathbb{R}$ be continuous, locally bounded uniformly in $n$, and degenerate elliptic. Define

$$
\begin{aligned}
F^{+}(t, x, p, X)=\lim _{m \rightarrow \infty} \sup \left\{F_{n}(s, y, q, Y): n \geq m,\right. \\
\qquad|t-s|+\|x-y\|+\|p-q\|+\|X-Y\| \leq 1 / m\}
\end{aligned}
$$

and

$$
\begin{aligned}
F_{-}(t, x, p, X)= & \lim _{m \rightarrow \infty} \inf \left\{F_{n}(s, y, q, Y): n \geq m,\right. \\
& |t-s|+\|x-y\|+\|p-q\|+\|X-Y\| \leq 1 / m\} .
\end{aligned}
$$

Let $A_{n}$ be linear, maximal monotone operators in $H$ such that $D\left(A^{*}\right) \subset$ $D\left(A_{n}^{*}\right)$ and

$$
\left\langle A_{n}^{*} x, x\right\rangle \geq \lambda_{n}\|x\|_{1}^{2} \quad \text { for } x \in D\left(A_{n}^{*}\right),
$$

where $\liminf _{n \rightarrow \infty} \lambda_{n} \geq \lambda$. Assume moreover that

$$
\text { if } \quad x_{n} \rightarrow x, A^{*} x_{n} \rightarrow A^{*} x \text { then } A_{n}^{*} x_{n} \rightarrow A^{*} x,
$$

and that for every test function $\varphi$, the family $A_{n}^{*} D \varphi$ is locally uniformly bounded. We then have the following theorem. 
THEOREM 6.1. Let the assumptions of this section be satisfied and let $B$ be compact. Let $u_{n}$ be locally uniformly bounded viscosity subsolutions (respectively, supersolutions) of

$$
u_{n}+\left\langle A_{n} x, D u_{n}\right\rangle+F_{n}\left(x, D u_{n}, D^{2} u_{n}\right)=0 \quad \text { in } H
$$

in the sense of Definition 3.2. Then the function

$$
u^{+}(x)=\lim _{m \rightarrow \infty} \sup \left\{u_{n}(y): n \geq m,\|x-y\| \leq 1 / m\right\}
$$

(respectively,

$$
\left.u_{-}(x)=\lim _{m \rightarrow \infty} \inf \left\{u_{n}(y): n \geq m,\|x-y\| \leq 1 / m\right\}\right)
$$

is a viscosity subsolution (respectively, supersolution) of

$$
u^{+}+\left\langle A x, D u^{+}\right\rangle+F_{-}\left(x, D u^{+}, D^{2} u^{+}\right)=0 \quad \text { in } H
$$

(respectively,

$$
\left.u_{-}+\left\langle A x, D u_{-}\right\rangle+F^{+}\left(x, D u_{-}, D^{2} u_{-}\right)=0 \quad \text { in } H\right)
$$

in the sense of Definition 3.4.

Notice that $u^{+}$does not have to be $B$-upper semicontinuous.

Proof. Let $\left(u^{+}-h(\|\cdot\|)\right)^{*}-\varphi$ have a local maximum equal to 0 at $x$. In light of Lemma 3.6 and local uniform boundedness of the $u_{n}$ we can assume that the maximum is global, strict in the $\|\cdot\|_{-1}$ norm, and such that

$$
u^{+}(y)-h(\|y\|),\left(u^{+}-h(\|\cdot\|)\right)^{*}(y)-\varphi(y) \rightarrow-\infty,
$$

and

$$
u_{n}(y)-h(\|y\|)-\varphi(y) \rightarrow-\infty
$$

as $\|y\| \rightarrow \infty$, uniformly in $n$. Then there must exist a sequence $x_{n}$ such that $\left\|x_{n}-x\right\|_{-1} \rightarrow 0,\left\|x_{n}\right\| \leq C$, and

$$
u^{+}\left(x_{n}\right)-h\left(\left\|x_{n}\right\|\right)-\varphi\left(x_{n}\right) \geq-1 / n .
$$

Therefore there exist $y_{n}$ and $m_{n}$ such that

$$
u_{m_{n}}\left(y_{n}\right)-h\left(\left\|y_{n}\right\|\right)-\varphi\left(y_{n}\right) \geq-2 / n .
$$

Let $z_{n}$ be a global maximum of $u_{m_{n}}(y)-h(\|y\|)-\varphi(y)$. It exists because of the decay of this function at infinity and the fact that, because $B$ is compact, $B$ upper semicontinuity is equivalent to weak sequential upper semicontinuity. Obviously $\left\|z_{n}\right\| \leq C_{1}$ and we also have

$$
\begin{aligned}
u_{m_{n}}\left(z_{n}\right)+\lambda_{m_{n}}\left\|z_{n}\right\|_{1}^{2} \frac{h^{\prime}\left(\left\|z_{n}\right\|\right)}{\left\|z_{n}\right\|} & +\left\langle z_{n}, A_{m_{n}}^{*} D \varphi\left(z_{n}\right)\right\rangle \\
& +F_{m_{n}}\left(z_{n}, D \psi\left(z_{n}\right), D^{2} \psi\left(z_{n}\right)\right) \leq 0,
\end{aligned}
$$

where $\psi(y)=h(\|y\|)-\varphi(y)$. Using the boundedness of $z_{n}$, the fact that $h^{\prime}(\|y\|) /\|y\|>c>0$ for $\|y\| \leq C_{1}$, and the local uniform boundedness of the 
$F_{n}$ and $A_{m_{n}}^{*} D \varphi$, we hence obtain $\left\|z_{n}\right\|_{1} \leq C_{2}$, which implies that $z_{n} \rightarrow z$ in $H_{1}$ and $z_{n} \rightarrow z$ in $H$ for some $z \in H_{1}$.

Therefore $u^{+}(z) \geq \lim \sup _{n \rightarrow \infty} u_{m_{n}}\left(z_{n}\right)$, which yields $u^{+}(z)-h(\|z\|)-$ $\varphi(z) \geq 0$, i.e. $z=x$. Moreover it also follows that

$$
\lim _{n \rightarrow \infty} u_{m_{n}}\left(z_{n}\right)=\left(u^{+}-h(\|\cdot\|)\right)^{*}(x)+h(\|x\|) .
$$

We can now pass to liminf in (6.5) to conclude the proof.

The following theorem is an immediate corollary of Theorems 6.1 and 4.1.

THEOREM 6.2. Let A satisfy (2.1) and (2.2). Let the assumptions of this section on $A_{n}$ and $F_{n}$ be satisfied, and let $B$ be compact. Let $F_{-}=F^{+}=: F$ satisfy the assumptions of Theorem 4.1. Let $u_{n}$ be locally bounded (uniformly in $n$ ) viscosity solutions of (6.3) in the sense of Definition 3.2. Let $u^{+}$and $-u_{-}$be bounded from above. Then $u^{+}=u_{-}=: u, u$ is locally uniformly $B$-continuous (i.e. $u$ is weakly sequentially continuous), and $u$ is the unique bounded viscosity solution of (1.1) in the sense of Definition 3.2. Moreover the functions $u_{n}$ converge to $u$ pointwise as $n \rightarrow \infty$ and the convergence is uniform on bounded subsets of $H_{\alpha}$ for every $\alpha>0$.

We point out that the limiting Hamiltonians $F^{+}$and $F^{-}$may be of first order so the above theorems can be applied to singular perturbation problems and small noise limits.

The time dependent version of Theorem 6.2 is the following. The functions $u^{+}$and $u^{-}$below are now defined by taking lim sup and lim inf in both variables $s$ and $y$.

TheOREM 6.3. Let A satisfy (2.1) and (2.2). Let the assumptions of this section on $A_{n}$ and $F_{n}$ be satisfied, and let $B$ be compact. Let $F_{-}=F^{+}=: F$ and let $F$ and $g$ satisfy the assumptions of Theorem 4.4. Let $u_{n}$ be viscosity solutions of

$$
\left(u_{n}\right)_{t}+\left\langle A_{n} x, D u_{n}\right\rangle+F_{n}\left(t, x, D u_{n}, D^{2} u_{n}\right)=0 \quad \text { in }(0, T) \times H
$$

in the sense of Definition 3.3 and suppose that

$$
\begin{gathered}
\left|u_{n}(x)\right| \leq C\left(1+\|x\|^{k}\right) \quad \text { for some } C>0, \\
\lim _{t \rightarrow 0}\left|u_{n}(t, x)-g(x)\right|=0 \quad \text { uniformly on bounded sets, }
\end{gathered}
$$

uniformly in $n$. Then $u^{+}=u_{-}=: u, u$ is weakly sequentially continuous, and $u$ is the unique viscosity solution of (1.2) in the sense of Definition 3.3 satisfying (6.6) and (6.7). Moreover the functions $u_{n}$ converge to $u$ pointwise as $n \rightarrow \infty$ and the convergence is uniform on bounded subsets of $\left[0, T_{1}\right] \times H_{\alpha}$ for every $\alpha>0$ and $0<T_{1}<T$.

We close this section by showing how half-relaxed limits can be applied to proving convergence of finite-dimensional approximations. 
Denote by $V_{N}$ the space spanned by the eigenvectors of $B$ corresponding to the eigenvalues that are greater than or equal to $1 / N$. Let $P_{N}$ be the orthogonal projection in $H$ onto $V_{N}$. Define

$$
A_{N}=\left(P_{N} A^{*} P_{N}\right)^{*}, \quad B_{N}=B P_{N} .
$$

Then $A_{N}$ is bounded and monotone in $H, A_{N}$ and $B_{N}$ satisfy (2.1), and

$$
\left\langle A_{N}^{*} x, x\right\rangle \geq \lambda\left\|P_{N} x\right\|_{1}^{2} \quad \text { for } x \in H .
$$

In what follows we denote $Q_{N}=I-P_{N}$. We now have the following result.

Theorem 6.4. Let A satisfy (2.1), (2.2), and let $D\left(A^{*}\right)=R(B)$. Let $F$ satisfy $(\mathbf{1})_{0},(\mathbf{2})$ with $\lambda B Q_{N}$ replaced by $\lambda Q_{N},(\mathbf{3})$, let $\sup _{x \in H}|F(x, 0,0)|=$ $M<\infty$, and let $B$ be compact. Let $u$ be the unique bounded viscosity solution of $(1.1)$, and let $u_{N}(x)=v_{N}\left(P_{N} x\right)$, where the $v_{N}$ are the viscosity solutions of

$$
v_{N}+\left\langle A_{N} x, D v_{N}\right\rangle+F\left(x, D v_{N}, D^{2} v_{N}\right)=0 \quad \text { in } H_{N} .
$$

Then $u_{N} \rightarrow u$ pointwise in $H$ as $N \rightarrow \infty$ and the convergence is uniform on bounded subsets of $H_{\alpha}$ for every $\alpha>0$.

Proof. Under our assumptions equation (6.9) has a unique viscosity solution $v_{N}$ such that $\left|v_{N}\right| \leq M$ for every $N \geq 1$. Also (see [25]) the functions $u_{N}$ are viscosity solutions of

$$
u_{N}+\left\langle A_{N} x, D u_{N}\right\rangle+F\left(P_{N} x, P_{N} D u_{N}, P_{N} D^{2} u_{N} P_{N}\right)=0 \text { in } H .
$$

Since the above equations have only bounded terms the solutions can be interpreted in the usual sense of [20], which in particular implies that the $u_{N}$ are solutions in the sense of Definition 3.2.

We first observe that the $A_{N}$ satisfy (6.2) with strong convergence. This follows from the proof of Lemma 2.3 of [5] upon noticing that $D\left(A^{*}\right)=R(B)$ guarantees that the operator $Q=A^{*} B+c B$ has bounded inverse $Q^{-1}=$ $B^{-1}\left(A^{*}+c I\right)^{-1}$ for every $c>0$. This last statement is a trivial consequence of the closed graph theorem.

We next claim that for every test function $\varphi$, the family $A_{N}^{*} D \varphi$ is locally uniformly bounded. Indeed,

$$
\begin{aligned}
& \left\|A_{N}^{*} D \varphi(x)\right\| \leq\left\|A^{*} B P_{N} B^{-1}\left(A^{*}+I\right)^{-1}\left(A^{*}+I\right) D \varphi(x)\right\| \\
& \quad \leq\left\|A^{*} B\right\|\left\|B^{-1}\left(A^{*}+I\right)^{-1}\right\|\left\|\left(A^{*}+I\right) D \varphi(x)\right\| \leq C\left\|\left(A^{*}+I\right) D \varphi(x)\right\| .
\end{aligned}
$$

However, we cannot invoke Theorem 6.1 directly as the $A_{N}$ only satisfy (6.8). Instead, we follow its proof pointing out the main differences. Repeating the previous arguments we now deduce, instead of (6.4), that there exist $y_{N}$ and $m_{N}$ such that

$$
u_{m_{N}}\left(P_{m_{N}} y_{N}\right)-h\left(\left\|P_{m_{N}} y_{N}\right\|\right)-\varphi\left(P_{m_{N}} y_{n}\right) \geq-2 / N .
$$


We then take $z_{N}$ to be a global maximum of $v_{m_{N}}(y)-h(\|y\|)-\varphi(y)$ over $H_{m_{N}}$. As before obviously $\left\|z_{N}\right\| \leq C_{1}$ and (6.5) becomes

$$
\begin{aligned}
v_{m_{N}}\left(z_{N}\right)+\lambda & \left\|z_{N}\right\|_{1}^{2} \frac{h^{\prime}\left(\left\|z_{N}\right\|\right)}{\left\|z_{N}\right\|}+\left\langle z_{N}, A_{m_{N}}^{*} D \varphi\left(z_{N}\right)\right\rangle \\
& +F_{m_{n}}\left(z_{N}, P_{m_{N}} D \psi\left(z_{N}\right), P_{m_{N}} D^{2} \psi\left(z_{N}\right) P_{m_{N}}\right) \leq 0,
\end{aligned}
$$

where $\psi(y)=h(\|y\|)-\varphi(y)$. This implies that (notice that $z_{N}=P_{m_{N}} z_{N}$ ) $\left\|z_{N}\right\|_{1} \leq C_{2}$, which gives $z_{N} \rightarrow z$ in $H_{1}$ and $z_{N} \rightarrow z$ in $H$ for some $z \in H_{1}$. Therefore $u^{+}(z) \geq \lim \sup _{N \rightarrow \infty} u_{m_{N}}\left(z_{N}\right)=\lim \sup _{N \rightarrow \infty} v_{m_{N}}\left(z_{N}\right)$ and this gives $u^{+}(z)-h(\|z\|)-\varphi(z) \geq 0$, i.e. $z=x$. We also obtain

$$
\lim _{N \rightarrow \infty} u_{m_{N}}\left(z_{N}\right)=\left(u^{+}-h(\|\cdot\|)\right)^{*}(x)+h(\|x\|) .
$$

We can now pass to liminf in (6.10) using Lemma 2.8 of [25].

In spite of the novelty of the method of half-relaxed limits in infinite dimensions, Theorem 6.4 is not really new under our assumptions. Convergence of finite-dimensional approximations was proved in [25] (following a similar method for first order equations of [5]) by first proving uniform continuity estimates for the $u_{N}$ and then showing their local uniform convergence. In [25] assumption (2.2) was not needed but here condition (2) is a little more general. However, our new method may succeed in situations where we may not be able to obtain uniform a priori estimates for the continuity of $u_{N}$.

Similar results can also be obtained for time dependent problems and for problems where we do not assume that $D\left(A^{*}\right)=R(B)$. We do not work out the details here as they are technical and lengthy, and the final statements are similar to the results of [25]. However, there is a significant difference between the approximations used in [25] (and in [5] before) and the ones we would need here for the half-relaxed limits. In [25] the operator $A$ was first approximated by its Yosida approximation $A_{\lambda}$ and then by $A_{\lambda, N}=$ $P_{N} A_{\lambda} P_{N}$. We do not know if this process would succeed here. It seems that we need first to take $A_{\lambda}=A+\lambda B^{-1}$ and then use $A_{\lambda, N}=P_{N} A_{\lambda} P_{N}$ for the above $A_{\lambda}$. This kind of approximation procedure was used in [6] and we refer the readers to this paper for some ideas and hints on how the proof should proceed in our case.

\section{References}

[1] M. Arisawa, H. Ishii and P. L. Lions, A characterization of the existence of solutions for Hamilton-Jacobi equations in ergodic control problems with applications, Appl. Math. Optim. 42 (2000), 35-50.

[2] P. Cannarsa and M. E. Tessitore, Infinite dimensional Hamilton-Jacobi equations and Dirichlet boundary control problems of parabolic type, SIAM J. Control Optim. 34 (1996), 1831-1847. 
[3] M. G. Crandall, H. Ishii and P. L. Lions, User's guide to viscosity solutions of second order partial differential equations, Bull. Amer. Math. Soc. 27 (1992), 1-67.

[4] M. G. Crandall, M. Kocan and A. Święch, On partial sup-convolutions, a lemma of P. L. Lions and viscosity solutions in Hilbert spaces, Adv. Math. Sci. Appl. 3 $(1993 / 4), 1-15$.

[5] M. G. Crandall and P. L. Lions, Hamilton-Jacobi equations in infinite dimensions, Part IV. Unbounded linear terms, J. Funct. Anal. 90 (1990), 237-283.

[6] - - - Hamilton-Jacobi equations in infinite dimensions, Part V. B-continuous solutions, ibid. 97 (1991), 417-465.

[7] - - - Hamilton-Jacobi equations in infinite dimensions, Part VI. Nonlinear A and Tataru's method refined, in: Evolution Equations, Control Theory, and Biomathematics (Han-sur-Lesse, 1991), P. Clément and G. Lumer (eds.), Lecture Notes in Pure and Appl. Math. 155, Dekker, New York, 1994, 51-89.

[8] -, 一, Hamilton-Jacobi equations in infinite dimensions. Part VII: The HJB equation is not always satisfied, J. Funct. Anal. 125 (1994), 111-148.

[9] G. Da Prato and J. Zabczyk, Second Order Partial Differential Equations in Hilbert Spaces, London Math. Soc. Lecture Note Ser. 293, Cambridge Univ. Press, Cambridge, 2002.

[10] J. Feng, Large deviation for a stochastic Cahn-Hilliard equation, Methods Funct. Anal. Topology 9 (2003), 333-356.

[11] - Large deviations for diffusions and Hamilton-Jacobi equations in Hilbert spaces, Ann. Probab. 34 (2006), 321-385.

[12] J. Feng and T. Kurtz, Large deviations for stochastic processes, preprint, 2005.

[13] F. Gozzi, E. Rouy and A. Święch, Second order Hamilton-Jacobi equations in Hilbert spaces and stochastic boundary control, SIAM J. Control Optim. 38 (2000), 400-430.

[14] F. Gozzi, S. S. Sritharan and A. Święch, Viscosity solutions of dynamic programming equations for the optimal control of the two-dimensional Navier-Stokes equations, Arch. Ration. Mech. Anal. 163 (2002), 295-327.

[15] - - - - Bellman equations associated to the optimal feedback control of stochastic Navier-Stokes equations, Comm. Pure Appl. Math. 58 (2005), 671-700.

[16] F. Gozzi and A. Święch, Hamilton-Jacobi-Bellman equations for the optimal control of the Duncan-Mortensen-Zakai equation, J. Funct. Anal. 172 (2000), 466-510.

[17] H. Ishii, Viscosity solutions for a class of Hamilton-Jacobi equations in Hilbert spaces, ibid. 105 (1992), 301-341.

[18] - Viscosity solutions of nonlinear second-order partial differential equations in Hilbert spaces, Comm. Partial Differential Equations 18 (1993), 601-651.

[19] D. Kelome, Viscosity solutions of second order equations in a separable Hilbert space and applications to stochastic optimal control, Ph.D. thesis, 2002.

[20] P. L. Lions, Viscosity solutions of fully nonlinear second-order equations and optimal stochastic control in infinite dimensions. Part III: Uniqueness of viscosity solutions for general second order equations, J. Funct. Anal. 86 (1989), 1-18.

[21] J. L. Lions and E. Magenes, Non-Homogeneous Boundary Value Problems and Applications, Vol. I, Springer, New York, 1972.

[22] M. Renardy, Polar decomposition of positive operators and a problem of Crandall and Lions, Appl. Anal. 57 (1995), no. 3-4, 383-385.

[23] K. Shimano, A class of Hamilton-Jacobi equations with unbounded coefficients in Hilbert spaces, Appl. Math. Optim. 45 (2002), 75-98.

[24] C. Stegall, Optimization of functions on certain subsets of Banach spaces, Math. Ann. 236 (1978), 171-176. 
[25] A. Święch, Unbounded second order partial differential equations in infinite dimensional Hilbert spaces, Comm. Partial Differential Equations 19 (1994), 1999-2036.

[26] —, Risk-sensitive control and differential games in infinite dimensions, Nonlinear Anal. 50 (2002), 509-522.

[27] D. Tataru, Viscosity solutions for Hamilton-Jacobi equations with unbounded nonlinear terms, J. Math. Anal. Appl. 163 (1992), 345-392.

[28] -, Viscosity solutions for Hamilton-Jacobi equations with unbounded nonlinear term - a simplified approach, J. Differential Equations 111 (1994), 123-146.

Department of Mathematics and Statistics

School of Mathematics

University of Massachusetts

Amherst, MA 01003, U.S.A.

Georgia Institute of Technology

Atlanta, GA 30332, U.S.A.

Current address:

E-mail: swiech@math.gatech.edu

Department of Mathematics and Statistics

McGill University

805 Sherbrooke St West

Montreal, QC, Canada H3A-2K6

E-mail: kelome@math.mcgill.ca

Received November 25, 2005

Revised version September 18, 2006 